\title{
Identification techniques for highly boosted W bosons that decay into hadrons
}

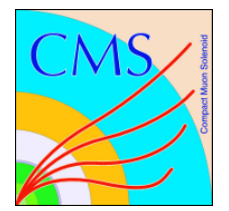

\section{The CMS collaboration}

E-mail: cms-publication-committee-chair@cern.ch

ABSTRACT: In searches for new physics in the energy regime of the LHC, it is becoming increasingly important to distinguish single-jet objects that originate from the merging of the decay products of $\mathrm{W}$ bosons produced with high transverse momenta from jets initiated by single partons. Algorithms are defined to identify such $\mathrm{W}$ jets for different signals of interest, using techniques that are also applicable to other decays of bosons to hadrons that result in a single jet, such as those from highly boosted $\mathrm{Z}$ and Higgs bosons. The efficiency for tagging $\mathrm{W}$ jets is measured in data collected with the CMS detector at a center-of-mass energy of $8 \mathrm{TeV}$, corresponding to an integrated luminosity of $19.7 \mathrm{fb}^{-1}$. The performance of $\mathrm{W}$ tagging in data is compared with predictions from several Monte Carlo simulators.

KEYworDs: Jets, Jet physics, Hadron-Hadron Scattering

ARXIV EPRINT: 1410.4227 


\section{Contents}

1 Introduction 1

2 CMS detector $\quad 2$

3 Event reconstruction $\quad 2$

4 Data and simulated event samples 3

4.1 Event topologies 3

4.2 Data and simulated event samples 4

4.3 Event selection 5

5 Algorithms for $\mathrm{W}$ jet identification $\quad 6$

$\begin{array}{lll}5.1 & \text { Substructure observables } & 7\end{array}$

$\begin{array}{ll}5.2 \text { Comparison of algorithms } & 10\end{array}$

$\begin{array}{lll}5.3 & \text { Performance in simulation } & 12\end{array}$

$\begin{array}{lll}5.4 & \mathrm{~W} \text {-polarization and quark-gluon composition } & 14\end{array}$

6 Performance in data and systematic uncertainties $\quad \mathbf{1 5}$

$\begin{array}{ll}\text { 6.1 Comparison of data and simulation } & 15\end{array}$

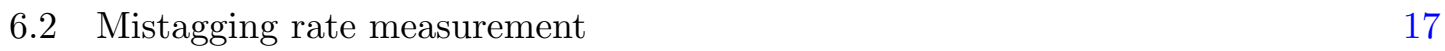

6.3 Efficiency scale factors and mass scale/resolution measurement 18

$\begin{array}{lll}6.4 & \text { Systematic uncertainties } & 20\end{array}$

$\begin{array}{lll}7 & \text { Summary and outlook } & 21\end{array}$

$\begin{array}{lr}\text { The CMS collaboration } & 29\end{array}$

\section{Introduction}

The LHC at CERN probes a new energy regime in particle physics, where searches for physics beyond the standard model (SM) at high mass scale often involve objects with large transverse momenta $\left(p_{\mathrm{T}}\right)$. In final states that contain the $\mathrm{W}^{ \pm}$and $\mathrm{Z}$ gauge bosons or Higgs bosons $(\mathrm{H})$, it is possible to achieve a high selection efficiency through the use of hadronic decay channels. At sufficiently large boost above order of $p_{\mathrm{T}}>200 \mathrm{GeV}$, the final state hadrons from the $\mathrm{W} \rightarrow \overline{\mathrm{q}} \mathrm{q}^{\prime}$ decay merge into a single jet, and the traditional analysis techniques relying on resolved jets are no longer applicable. However, in such cases the analysis of jet substructure can be used to identify those jets arising from decays of $\mathrm{W}$, $\mathrm{Z}$ or $\mathrm{H}$ bosons. Because the values of the mass of the $\mathrm{W}$ and $\mathrm{Z}$ bosons are rather close to each other, we do not distinguish the two, and refer to such jets collectively as $\mathrm{V}$ jets, while the Higgs boson mass is significantly higher and can be distinguished. The focus 
of this paper is solely on the identification of $\mathrm{W}$ jets, however, we note that many of the procedures described are equally applicable for handling highly boosted $\mathrm{Z}$ and $\mathrm{H}$ bosons.

Measurements of jet substructure observables related to identification of $\mathrm{W}$ bosons have been previously reported by CMS $[1,2]$ and ATLAS $[3,4]$. Several searches at CMS have employed jet substructure techniques for identifying ("tagging") W jets and Z jets. These include searches in all-jet t $\bar{t}$ final states [5, 6], single and pair produced $\mathrm{V}$ bosons in inclusive dijet final states [7, 8], and searches in the VV final states, where one of the vector bosons decays leptonically $[9,10]$. In these searches, a variety of different observables have been used to identify the $\mathrm{V}$ jets. This paper aims to compare and measure the performance in $8 \mathrm{TeV}$ pp collisions of various jet substructure techniques that can be used to distinguish $\mathrm{V}$ jets from more ordinary quark- and gluon-initiated jets, which we refer to as QCD jets.

This paper is organized as follows. The CMS detector is described in section 2. The procedures chosen for the reconstruction of events are described in section 3 . The data and simulated events used in our studies as well as the event selection criteria are presented in section 4. In section 5, through Monte Carlo (MC) simulation, we investigate the performance of jet substructure observables used to identify $\mathrm{W}$ jets, in order to find the best discriminants for such events. We compare these observables in different kinematic regimes, and examine factors that contribute to their performance. Their distributions in data are compared to those in $\mathrm{MC}$ simulations in section 6, to learn how well current MC simulations can model the physical processes responsible for jet substructure. The methods used to extract data-to-simulation scale factors needed to correct $\mathrm{W}$ boson tagging efficiencies obtained from MC simulation are discussed in section 6 , and the mistagging rate of QCD jets in data is extracted. The goal being to provide these as reference tools for analyzing events with jets from $\mathrm{V}$ bosons in the final state. Finally, we give a summary of our studies in section 7 .

\section{CMS detector}

The central feature of the CMS detector is a $3.8 \mathrm{~T}$ superconducting solenoid of $6 \mathrm{~m}$ internal diameter. A complex silicon tracker, a crystal electromagnetic calorimeter (ECAL), and a hadron calorimeter (HCAL) are located within the magnetic field volume. A muon system is installed outside the solenoid, and embedded in the steel return yoke. The CMS tracker consists of 1440 silicon pixel and 15148 silicon strip detector modules. The ECAL consists of 75848 lead tungstate crystals, which provide coverage in pseudorapidity of $|\eta|<1.48$ in the central barrel region and $1.48<|\eta|<3.00$ in the two forward endcap regions. The muon system includes barrel drift tubes covering the pseudorapidity range $|\eta|<1.2$, endcap cathode strip chambers $(0.9<|\eta|<2.5)$, and resistive plate chambers $(|\eta|<1.6)$. A more detailed description of the CMS detector, together with a definition of the coordinate system used and the relevant kinematic variables, can be found in ref. [11].

\section{Event reconstruction}

Jets are reconstructed by clustering particles obtained using the particle flow (PF) algorithm [12-14]. The PF procedure identifies each individual particle (a PF candidate) 
through an optimized combination of all subdetector information. The energy of photons is obtained directly from the ECAL measurement, corrected for suppression effects of energies from calorimetric channels with small signals (referred to as zero-suppression) [15]. The energy of an electron is determined from a combination of the track momentum at the main interaction vertex, the corresponding ECAL cluster energy, and the energy sum of all bremsstrahlung photons associated with the track. The energy of a muon is obtained from the corresponding track momentum. The energy of a charged hadron is determined from a combination of the track momentum and the corresponding ECAL and HCAL energies, corrected for zero-suppression effects, and calibrated for the nonlinear response of the calorimeters. Finally, the energy of a neutral hadron is obtained from the calibrated energies in ECAL and HCAL.

The PF candidates are clustered into jets using two algorithms: the anti- $k_{\mathrm{T}}$ algorithm [16] with the distance parameter $R=0.5$ (AK5), and the Cambridge-Aachen algorithm $[17,18]$ with the distance parameter $R=0.8$ (CA8), as implemented in FASTJET version 3.0.1 [19]. While the CA8 algorithm with a larger distance parameter is used throughout this paper to select and identify W jets, the AK5 algorithm is used to put requirements on additional QCD jets in the event selection. The choice of these algorithms is further explained in section 5. To mitigate the effect of multiple interactions in the same bunch crossing, the so-called pileup (PU), charged hadrons that are not associated with the primary vertex are removed from the list of PF candidates. The procedure is referred to as charged-hadron subtraction [20] and strongly reduces the dependence of the jet energy and substructure reconstruction on pileup. An event-by-event jet-area-based correction [21-23] is applied to remove the remaining energy due to neutral particles originating from the other pp collision vertices. All jet substructure observables are computed using PF candidates calibrated prior to jet clustering. However, the resulting jets require another small correction to the jet momentum and energy that accounts for tracking inefficiencies and threshold effects. The typical jet energy resolution is $5-10 \%$ for jets with $p_{\mathrm{T}}>200 \mathrm{GeV}$.

Two algorithms are used to reconstruct muons [24]: one proceeds from the inner tracker outwards, while the other starts from tracks measured in the muon chambers and matches them to those reconstructed in the silicon tracker. Muons are identified using selection criteria optimized for high- $p_{\mathrm{T}}$ muons [24]. The selected muon candidates must be isolated from charged hadron activity in the detector by requiring the scaler sum of transverse momenta $\left(I_{\mathrm{tk}}\right)$ of tracks within a cone of $\Delta R=\sqrt{(\Delta \phi)^{2}+(\Delta \eta)^{2}}<0.3$ around the muon track, divided by the muon $p_{\mathrm{T}}$, to be $I_{\mathrm{tk}} / p_{\mathrm{T}}<0.1$. Electrons are reconstructed using a Gaussian-sum filter algorithm [15, 25], and each electron candidate must furthermore pass the identification and isolation criteria optimized for high $p_{\mathrm{T}}$ electrons [25].

\section{Data and simulated event samples}

\subsection{Event topologies}

This study aims to distinguish $\mathrm{W}$ jets from QCD jets. We use three different final state topologies to establish $\mathrm{W}$ jet identification in a broad region of phase space, thereby en-

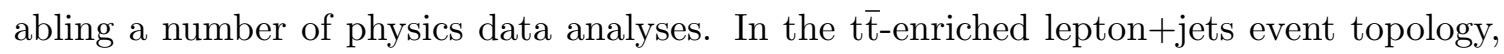


the decay of two top quarks results in a final state with two b quarks and two $\mathrm{W}$ bosons of which one decays leptonically and the other decays to hadrons. This topology provides a relatively pure source of $\mathrm{W}$ jets in data, and is used to compare the efficiencies of $\mathrm{W}$ tagging in data and in simulation. In contrast, the $\mathrm{W}+$ jet event topology, where the $\mathrm{W}$ boson decays leptonically, and the inclusive dijet event topology are used as a source of QCD jets to study their $\mathrm{W}$-jet tagging properties in data and in simulation. These are the benchmark scenarios for searches, where the leading backgrounds are SM W+jets and dijet production. The $\mathrm{W}+$ jet sample accesses the low $p_{\mathrm{T}}$ regime, while the dijet sample reaches higher $p_{\mathrm{T}}$, and therefore both samples are explored. To study the discrimination of $\mathrm{W}$ jets and QCD jets in the $\mathrm{W}+$ jet and dijet topologies, we use simulated samples of beyond-SM resonances decaying to the $\mathrm{WW}$ final state as source of $\mathrm{W}$ jets.

\subsection{Data and simulated event samples}

The data were collected with the CMS detector at a proton-proton (pp) center-of-mass energy of $8 \mathrm{TeV}$ and correspond to an integrated luminosity of $19.7 \pm 0.5 \mathrm{fb}^{-1}[26]$.

As the default simulated signal sample, we consider a resonance $\mathrm{X}$ that decays to a pair of longitudinally polarized $\mathrm{W}$ bosons. Such samples are produced by considering either a warped extra-dimensional model, where the SM fields propagate in the bulk [27-29], or models with SM-like high mass H bosons. Graviton resonance samples in the extra-dimensional model are produced with the JHUGEN 3.1.8 [30, 31], interfaced with PYTHIA 6 [32] for parton showering including the effect of hard gluon radiation. PYTHIA 6.426 is used with Tune Z2* [33] in this paper. SM-like H boson samples are produced with POWHEG 1.0 [34-36] interfaced with PYTHIA 6. To study the effect of W boson polarization on the distributions of substructure variables, the model with the SM Higgslike couplings is compared to a model with a purely pseudoscalar $\mathrm{H}$ boson which yields only transversely polarized $\mathrm{W}$ bosons. These samples are produced with the JHUGEN and PYTHIA 6 , with a resonance width of $\approx 1 \%$ chosen to be narrower than the experimental resolution of $5-10 \%$.

The background is modeled using QCD multijet, W+jets, WW/WZ/ZZ, Drell-Yan $\left(\mathrm{q} \overline{\mathrm{q}} \rightarrow \mathrm{Z} / \gamma^{*} \rightarrow \ell \ell\right), \mathrm{t} \overline{\mathrm{t}}$, and single top quark MC simulation samples. Three QCD multijet samples are compared. A first sample is generated with MADGRAPH v5.1.3.30 [37], with showering and hadronization performed with PYTHIA 6. The second sample is generated as well as evolved with HERWIG++ 2.5.0 [38] with tune version 23 [38]. The third sample is generated with Pythia 8.153 [39] with Tune 4C. MAdGraph, PYTHIA 6 and Pythia 8 are used with the CTEQ61L [40] parton distribution functions (PDF), while HERWIG++ is used with the MRST2001 [41] PDF. Two W+jets samples with different parton shower models are compared: one sample generated with MADGRAPH interfaced with PYTHIA 6

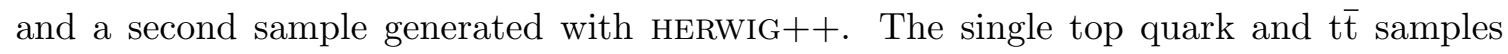
are simulated with POWHEG interfaced with PYTHIA 6 using the CT10 [42] PDF. An al-

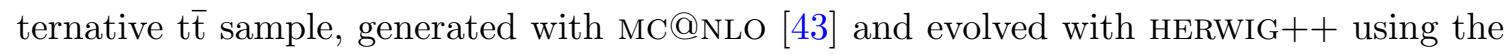
CTEQ6M [40] PDF, is also used for studies of systematic effects. The $\mathrm{Z}+$ jets process is simulated with MADGRAPH interfaced with PYTHIA 6. The VV production processes are simulated with PYTHIA 6 . 
All generated samples are processed through a GEANT4-based [44] simulation of the CMS detector. An average of 22 supplementary interactions are added to the generated events in order to match the additional particle production observed in data from the large number of PU proton-proton interactions occurring per LHC bunch crossing.

\subsection{Event selection}

The dijet and $\mathrm{W}+$ jet topologies are chosen to be in the kinematic regime typically considered in searches for new phenomena [7,9]. In both topologies we focus on the W-jet tagging properties of the highest $p_{\mathrm{T}} \mathrm{CA} 8$ jet in the event, requiring $|\eta|<2.4$, so that the core of the jet falls within the tracker acceptance. The ranges in jet $p_{\mathrm{T}}$ and the resonance masses $m_{\mathrm{X}}$ are chosen to have the $p_{\mathrm{T}}$ distributions similar for signal and for background. For the $\mathrm{W}+$ jet topology, the jet $p_{\mathrm{T}}$ is within $250-350 \mathrm{GeV}$ and $m_{\mathrm{X}}=600 \mathrm{GeV}$, while for the dijet topology, the jet $p_{\mathrm{T}}$ is within $400-600 \mathrm{GeV}$ and $m_{\mathrm{X}}=1 \mathrm{TeV}$.

Collision data events with a dijet final state are collected using the logical "OR" of a set of triggers based on requirements on $H_{\mathrm{T}}=\sum_{\text {jets }} p_{\mathrm{T}}$ (scalar sum of $p_{\mathrm{T}}$ of the AK5 jets), and on the invariant mass of the two jets of highest $p_{\mathrm{T}}$. Subsequent event selection follows closely the VV resonance search in ref. [7]. Events are initially selected by requiring at least two jets with $p_{\mathrm{T}}>30 \mathrm{GeV}$ and $|\eta|<2.4$. The two jets of highest $p_{\mathrm{T}}$ are required to have a pseudorapidity separation $|\Delta \eta|<1.3$, which rejects a large fraction of QCD multijet events. Finally, the dijet invariant mass is required to be larger than $890 \mathrm{GeV}$. This threshold is chosen such that the trigger selection for events with dijet masses above this threshold is $99 \%$ efficient. W-tagging is studied using the leading jet in the selected dijet events, with additional requirements set on jet $p_{\mathrm{T}}$.

The main goal of the kinematic selection of the $\mathrm{W}+$ jet sample is to isolate a sample of events with a highly boosted topology consistent with a leptonically decaying $\mathrm{W}$ boson

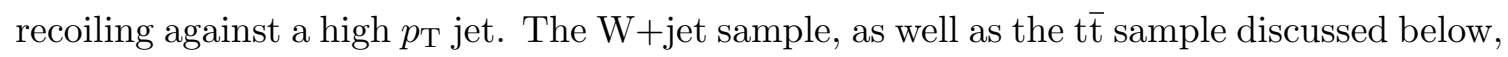
are collected using single-lepton triggers. The lepton $p_{\mathrm{T}}$ thresholds of these triggers are 40 and $80 \mathrm{GeV}$ for the muon and electron channels, respectively. Offline, at least one muon or one electron, with respective $p_{\mathrm{T}}>50 \mathrm{GeV}$ or $p_{\mathrm{T}}>90 \mathrm{GeV}$, is required within respective $|\eta|<2.1$ or $|\eta|<2.5$. Events containing additional muons with $p_{\mathrm{T}}>20 \mathrm{GeV}$ and $|\eta|<2.4$ or additional electrons with $p_{\mathrm{T}}>20 \mathrm{GeV}$ and $|\eta|<2.5$ are rejected, in order to improve the purity of $\mathrm{W}+$ jet events. A requirement on the imbalance in transverse momentum $\left(E_{\mathrm{T}}^{\text {miss }}\right)$ is used to reduce the QCD multijet background. The $E_{\mathrm{T}}^{\mathrm{miss}}$ is computed from the negative transverse component of the vector sum of all $\mathrm{PF}$ candidate momenta, and is required to be above $50 \mathrm{GeV}$ or $80 \mathrm{GeV}$ for the muon and electron channel. The threshold is higher in the electron channel to further suppress the larger background from multijet processes. The $p_{\mathrm{T}}$ of the leptonically decaying $\mathrm{W}$ boson and of the CA8 jet with highest $p_{\mathrm{T}}$, are required to be $>200 \mathrm{GeV}$. Additional criteria are applied to ensure that the leptonic $\mathrm{W}$ boson and the CA8 jet are mostly back-to-back in the transverse plane: $\Delta R$ between the lepton and the jet must be greater than $\pi / 2$; the azimuthal distance $\Delta \phi$ between $E_{\mathrm{T}}^{\text {miss }}$ and the jet must be greater than 2.0 radians; and the azimuthal distance $\Delta \phi$ between the leptonically decaying $\mathrm{W}$ boson and the $\mathrm{CA} 8$ jet must also be greater than 2.0 radians. Finally, a cutoff on additional jet activity in the event is applied to reduce the amount of 


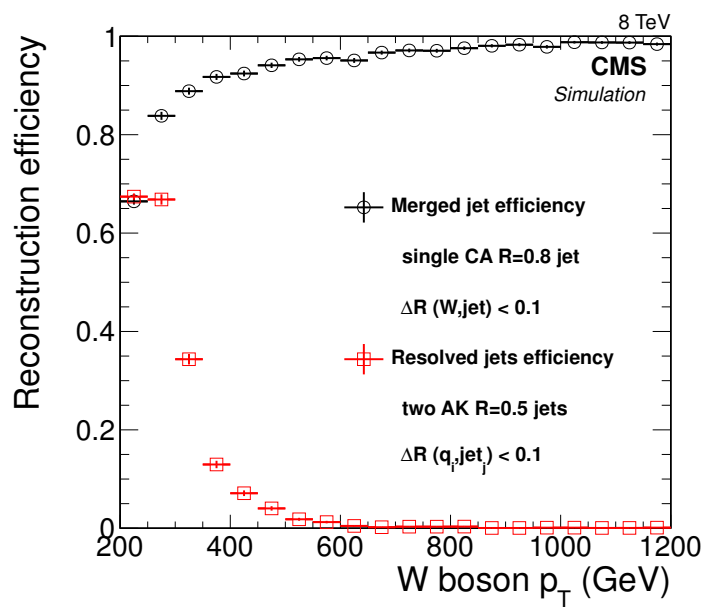

Figure 1. Efficiency to reconstruct a CA8 jet within $\Delta R<0.1$ of a generated $\mathrm{W}$ boson, and the efficiency to reconstruct two AK5 jets within $\Delta R<0.1$ of the generated quarks from longitudinally polarized $\mathrm{W}$ bosons, as a function of the $p_{\mathrm{T}}$ of the $\mathrm{W}$ boson.

$\mathrm{t} \overline{\mathrm{t}}$ background. We identify additional $\mathrm{b}$ jet candidates in the event by requiring that an AK5 jet, with an angular distance of $\Delta R>0.8$ to the CA8 jet, passes the CSV b-tagging discriminant [45] using a medium working point. To suppress t $\overline{\mathrm{t}}$ background in the $\mathrm{W}+$ jet selections described above, we require that no such $\mathrm{b}$ jets be present in the event.

To select the $t \bar{t}$ sample, we use the kinematic selection described above for the $\mathrm{W}+$ jet topology, but instead require that there is at least one AK5 b jet, with an angular distance of $\Delta R>0.8$ to the $\mathrm{CA} 8$ jet considered as $\mathrm{W}$ jet candidate. To increase the statistical precision of the sample, we select the CA 8 jet with the largest mass and with $\Delta \phi$ between the lepton and the jet greater than $\pi / 2$ as $\mathrm{W}$ jet candidate, rather than the highest $p_{\mathrm{T}}$ CA8 jet.

\section{Algorithms for $\mathrm{W}$ jet identification}

A jet clustering algorithm with $R=0.8$ is used to identify $\mathrm{W}$ jets. A large value of $R$ increases the efficiency to reconstruct $\mathrm{W}$ bosons with small boost as single jets, since the average angular distance between the $\mathrm{W}$ decay products is inversely proportional to the $p_{\mathrm{T}}$ of the $\mathrm{W}$. The chosen value of $R$ provides a high efficiency for $\mathrm{W}$ bosons with small boost and ensures that no efficiency is lost in the transition from classical $\mathrm{W}$ reconstruction from two small jets at low $\mathrm{W} p_{\mathrm{T}}$ and reconstruction from a single large jet at higher $\mathrm{W} p_{\mathrm{T}}$ (see e.g. ref. [46]). Another point to consider when choosing the value of $R$, is the $t \bar{t}$ data sample available for validating highly boosted $\mathrm{W}$ jets. If $R$ is chosen too large, the b quark from the $\mathrm{t} \rightarrow \mathrm{Wb}$ decay tends to merge into the $\mathrm{W}$ jet. The chosen value of $R$ is the result of a compromise between high efficiency for $\mathrm{W}$ bosons with small boost and a sufficiently large sample of $\mathrm{W}$ jets in $\mathrm{t} \overline{\mathrm{t}}$ data for validating the $\mathrm{W}$ jet identification algorithms.

Figure 1 shows the $p_{\mathrm{T}}$ range of $\mathrm{W}$ bosons for which the $R=0.8$ algorithm is efficient and compares this to the efficiency for reconstructing $\mathrm{W}$ bosons from two $R=0.5$ jets. Above a $p_{\mathrm{T}}$ of $200 \mathrm{GeV}$, the $\mathrm{CA} 8$ jet algorithm, used to identify $\mathrm{W}$ jets, becomes more 
efficient than the reconstruction of a $\mathrm{W}$ boson from two AK5 jets. In this paper we therefore study substructure observables to identify $\mathrm{W}$ jets for an $R=0.8$ algorithm. Whether an AK or a CA algorithm is used in such comparison does not affect the overall conclusion. The choice of CA (with $R=0.8$ ) and AK $(R=0.5)$ is simply due to their wide use in CMS publications, where CA was introduced in the first top tagging algorithm paper of CMS [47]. Whenever we refer to efficiency $(\epsilon)$ in this paper, we refer to the full efficiency to identify a $\mathrm{W}$ boson relative to all generated $\mathrm{W}$ bosons decaying to hadrons.

\subsection{Substructure observables}

As the mass of the $\mathrm{W}$ boson is larger than the mass of a typical QCD jet, the jet mass is the primary observable that distinguishes a $\mathrm{W}$ jet from a QCD jet. The bulk of the $\mathrm{W}$ jet mass arises from the kinematics of the two jet cores that correspond to the two decay quarks. In contrast, the QCD jet mass arises mostly from soft gluon radiation. For this reason, the use of jet grooming methods such as filtering [48], trimming [49], or pruning [50, 51], improves discrimination by removing the softer radiation, as this shifts the jet mass of QCD jets to smaller values, while maintaining the jet mass for $\mathrm{W}$ jets close to the $\mathrm{W}$ mass. Studies of these grooming methods have been performed in ref. [1], with the conclusion that the pruned jet mass provides the best separation between W signal and QCD background. In this paper, we use the grooming parameters proposed by the original authors.

Pruned jet mass: is obtained by removing the softest components of a jet. The CA 8 jet is reclustered from its original jet constituents, however the CA clustering sequence is modified to remove soft and wide-angle protojets (single particles, or groups of particles already combined in the previous steps). In each recombination step, its hardness $z$ is defined as $z=\min \left\{p_{\mathrm{T}}^{i}, p_{\mathrm{T}}^{j}\right\} / p_{\mathrm{T}}^{p}$, where $p_{\mathrm{T}}^{i}$ and $p_{\mathrm{T}}^{j}$ are the $p_{\mathrm{T}}$ of the two protojets to be combined and $p_{\mathrm{T}}^{p}$ is the $p_{\mathrm{T}}$ of the combination of the two protojets. The protojet with the lower $p_{\mathrm{T}}^{i}$ is ignored if $z<z_{\text {cut }}=0.1$, and if it forms an angle $\Delta R$ wider than $D_{\text {cut }}=m^{\text {orig }} / p_{\mathrm{T}}^{\text {orig }}$ relative to the axis of the combination of the two protojets, where $m^{\text {orig }}$ and $p_{\mathrm{T}}^{\text {orig }}$ are the mass and $p_{\mathrm{T}}$ of the original CA 8 jet. The pruned jet mass distributions for $\mathrm{W}$ jets and QCD jets are shown in figure 2 (upper left) at generator level and detector level with pileup. Comparing the generator level predictions for the pruned jet mass of $\mathrm{W}$ jets with those at detector level with pileup, the widening of the peak due to detector resolution can be observed.

Further discrimination between $\mathrm{W}$ and QCD jets can be obtained from a more extensive use of jet substructure. Here we consider the following observables.

Mass drop $\boldsymbol{\mu}$ [48]: is calculated from the two subjets that are obtained by undoing the last iteration of the $\mathrm{CA}$ jet clustering via pruning. The idea behind the mass drop is that the $\mathrm{W}$ jet is formed by merging the showers of two decay quarks, and thus the mass of each quark subjet is much smaller than the mass of the $\mathrm{W}$ jet. In contrast, a massive QCD jet is formed through continuous soft radiation; the subjet with larger mass contains the bulk of the jet and the ratio of the mass of the large subjet to the total mass is therefore close to unity. We define the mass drop $\mu$ as the ratio of the masses of the higher mass subjet $\left(m_{1}\right)$ and the total pruned jet $\left(m_{\text {jet }}\right)$. The two subjets can also be used to estimate 

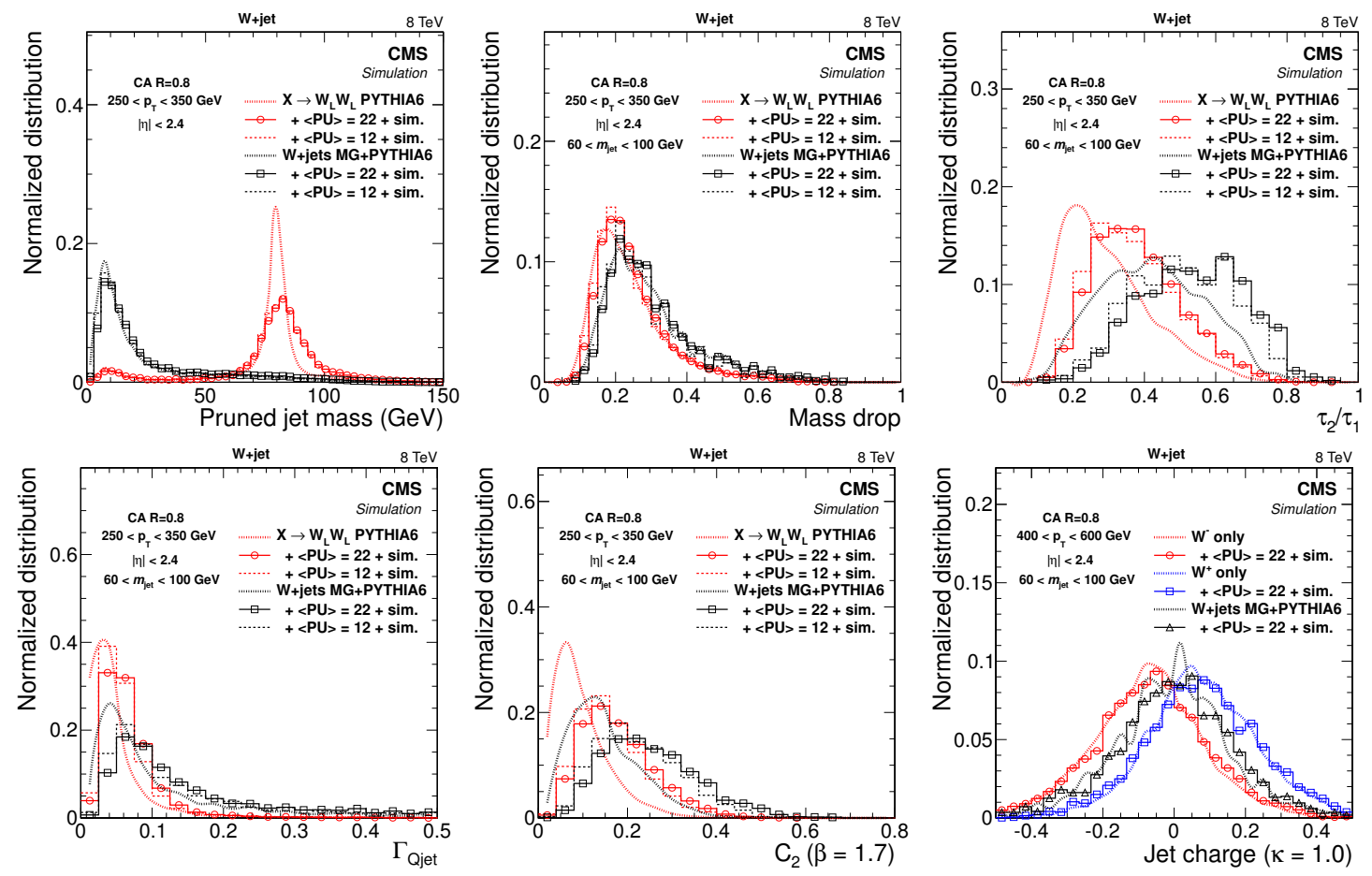

Figure 2. Distributions of six variables characterising jet substructure in simulated samples of highly boosted and longitudinally polarized $\mathrm{W}$ bosons and inclusive QCD jets expected in the $\mathrm{W}+$ jet topology. The discriminator distributions (except for the pruned jet mass in the upper left panel) are shown after a selection on the pruned jet mass of $60<m_{\text {jet }}<100 \mathrm{GeV}$. MG denotes the MADGRAPH generator. Thick dashed lines represent the generator predictions without pileup interactions and without CMS detector simulation. The histograms are the expected distributions after full CMS simulation with pileup corresponding to an average number of 12 and 22 interactions. (upper middle) gives the mass drop variable, (upper right) the $\mathrm{N}$-subjettiness ratio $\tau_{2} / \tau_{1}$, (lower left) the Qjet volatility, (lower middle) the energy correlation function double ratio $C_{2}^{\beta}$, and (lower left) the jet charge.

their $\Delta R$, which can provide additional discrimination. The distribution of $\mu$ is shown in figure 2 (upper middle). The differences between the generator level predictions and those at detector level with pileup are small for this observable, because the detector can resolve the two relatively well separated subjets.

N-subjettiness $\tau_{N}$ [52]: is a generalized jet shape observable. N-subjettiness is computed under the assumption that the jet has $\mathrm{N}$ subjets, and it is the $p_{\mathrm{T}}$-weighted $\Delta R$ distance between each jet constituent and its nearest subjet axis:

$$
\tau_{N}=\frac{1}{d_{0}} \sum_{k} p_{\mathrm{T}, k} \min \left\{\Delta R_{1, k}, \Delta R_{2, k}, \cdots, \Delta R_{N, k}\right\}
$$

where $k$ runs over all constituent particles. The normalization factor is $d_{0}=\sum_{k} p_{\mathrm{T}, k} R_{0}$ and $R_{0}$ is the original jet distance parameter. The $\tau_{N}$ observable has a small value if the jet is consistent with having $\mathrm{N}$ or fewer subjets, as almost every jet constituent will be close in $\Delta R$ to its own true subjet. For discrimination between $\mathrm{W}$ jets with two subjets and QCD 
jets consistent with corresponding to a single subjet, the ratio $\tau_{2} / \tau_{1}$ is particularly useful as it tends to smaller values for $\mathrm{W}$ jets. The subjet axes are obtained by running the exclusive $k_{\mathrm{T}}$ algorithm [53], and reversing the last $\mathrm{N}$ clustering steps. The axes can be optimized to minimize the N-subjettiness value. As default, we use a "one-pass" optimization of the exclusive $k_{\mathrm{T}}$ axes, where one step of the iterative optimization is performed. By default $\tau_{2} / \tau_{1}$ is calculated from the unpruned CA8 jets, but we also consider a pruned $\tau_{2} / \tau_{1}$ calculated from pruned CA 8 jets. Figure 2 (upper right) shows the $\tau_{2} / \tau_{1}$ distribution for $\mathrm{W}$ jets and QCD jets after requiring $60<m_{\text {jet }}<100 \mathrm{GeV}$, and demonstrates its discrimination power after the pruned jet mass selection. The distributions at detector level with pileup are shifted significantly compared to the generator level predictions, though the discrimination power is preserved. The shift was due equally to detector effects and pileup.

Qjet volatility $\boldsymbol{\Gamma}_{\text {Qjet }}[\mathbf{5 4}]$ : is a statistical measure of an ensemble of similar jet clustering sequences. A jet is defined by its cluster sequence, which is topologically a tree and is here referred to as "jet tree". By randomizing the recombination scheme and running the pruning algorithm for each jet tree, we can define a family of trees for each jet from which we can compute a distribution of jet masses. The continuous soft radiation that forms massive QCD jets results in clustering sequences susceptible to fluctuations - a small deviation in soft radiation can result in a very different order of putting the jet together. In contrast, W jets are characterized by two strong jet cores, and small perturbations usually yield nearly identical clustering sequences. Therefore a large volatility of the clustering sequence is a characteristic of QCD jets, and can be used to distinguish them from signal W jets.

The procedure for quantifying the volatility of the jet clustering sequence is as follows. At every step of clustering, a weight $w_{i j}$ is assigned to each constituent pair, and then one of the available pairs are randomly chosen and combined. The default weight is defined as:

$$
w_{i j}=\exp \left\{-\alpha \frac{d_{i j}-d^{\min }}{d^{\min }}\right\}
$$

where $d_{i j}=\Delta R_{i j}^{2}$ is the $(\eta, \phi)$ distance measure of the CA algorithm within the $i j$ pair, $d^{\text {min }}$ is its minimum over all pairs at this stage in the clustering, and $\alpha$ is the rigidity controlling the level of randomness, where for $\alpha \rightarrow \infty$ represents the limit of a classical jet algorithm. We choose to generate 50 random jet trees. Qjet volatility is defined as the root-mean-square (RMS) of the jet mass distribution, divided by the average jet mass, or $\Gamma_{\text {Qjet }}=\mathrm{RMS} /\langle m\rangle$. To improve the speed of the algorithm without greatly degrading the performance, before Qjet clustering we pre-cluster the jet constituents down to 35 protojets. Figure 2 (lower left) shows the distributions in $\Gamma_{\text {Qjet }}$.

Energy correlation function double ratio $C_{2}^{\beta}$ [55]: is defined as follows:

$$
C_{2}^{\beta}=\frac{\sum_{i<j<k} p_{\mathrm{T} i} p_{\mathrm{T} j} p_{\mathrm{T} k}\left(R_{i j} R_{i k} R_{j k}\right)^{\beta} \sum_{i} p_{\mathrm{T} i}}{\left(\sum_{i<j} p_{\mathrm{T} i} p_{\mathrm{T} j}\left(R_{i j}\right)^{\beta}\right)^{2}}
$$

where $i, j$ and $k$ runs over all constituent particles satisfying $i<j<k$. Similarly to the ratio $\tau_{2} / \tau_{1}$, the numerator quantifies how likely a jet is composed of two subjets, while 
the denominator gives a probability for being composed of one subjet. We study $C_{2}^{\beta}$ with $\beta=1.7$ as suggested in ref. [55], which is suited to discriminate two-prong $\mathrm{W}$ jets from QCD jets consistent with having a single subjet. The distribution of $C_{2}^{\beta}$ is given in figure 2 (lower middle).

Planar flow with $\boldsymbol{R}=\mathbf{0 . 5}$ and trimmed grooming sensitivity [56]: have also been considered in this study. Planar flow characterises the geometric distribution of energy deposition from a jet, which discriminates $\mathrm{W}$ jets from QCD jets, as the latter are more isotropic. Trimmed grooming sensitivity is defined as the decrease in jet mass, when the trimming algorithm [49] is applied to the jet.

Jet charge, $Q^{\kappa}[57]:$ is a measure of the electric charge of the parton that is the origin of the jet. This variable has a long history in flavor tagging of neutral B mesons, and it is defined as the $p_{\mathrm{T}}$-weighted average charge of the jet:

$$
Q^{\kappa}=\frac{\sum_{i} q_{i}\left(p_{\mathrm{T}}^{i}\right)^{\kappa}}{\left(p_{\mathrm{T}}^{\mathrm{jet}}\right)^{\kappa}}
$$

Here $i$ runs over all particles in a jet. Our default choice for $\kappa$ is 1 . It can be used to provide additional discrimination among quark jets, gluon jets and $\mathrm{W}$ jets or also to distinguish the charged W' signal from that of a neutral Z'. The differences between the jet charge distribution of $\mathrm{W}^{ \pm}$jets and of neutral jets can be seen in figure 2 (lower right). Detector resolution and pileup have almost no effect on this variable as it is built from charged hadrons identified using the tracker where those from PU vertices are discarded.

\subsection{Comparison of algorithms}

We compare the performance of observables used to identify $\mathrm{W}$ jets with the goal of establishing which provides the best signal-to-background discrimination between $\mathrm{W}$ jets and QCD jets. Because the pruned jet mass is the best discriminant, we examine the other variables only for jets satisfying $60<m_{\text {jet }}<100 \mathrm{GeV}$. Observables highly correlated with the pruned jet mass will therefore show weaker additional improvement in performance.

The figure of merit for comparing different substructure observables is the background rejection efficiency as a function of signal efficiency ("receiver operating characteristic", or the ROC curve). Figure 3 shows the performance of the observables in the $\mathrm{W}+$ jet final state for jet $p_{\mathrm{T}} 250-350 \mathrm{GeV}$. The pruned jet mass selection is applied in both the numerator and the denominator of the efficiency, and only the additional discrimination power of the other observables is therefore shown in the figure. The performance of the $\tau_{2} / \tau_{1}$, pruned $\tau_{2} / \tau_{1}$, exclusive- $k_{\mathrm{T}} \tau_{2} / \tau_{1}, \Gamma_{\text {Qjet }}, C_{2}^{\beta}$, mass drop, and jet charge are compared. For the jet charge ROC curve, a positively charged lepton is required in the event selection, and therefore the discrimination power of negatively charged $\mathrm{W}$ jets against QCD jets is compared. We find that the best performant variable is $\tau_{2} / \tau_{1}$ up to an efficiency of $75 \%$. Above an efficiency of $75 \%, \Gamma_{\text {Qjet }}$ is the best variable. The pruned $\tau_{2} / \tau_{1}$ is slightly worse than the default $\tau_{2} / \tau_{1}$. The performance of the $\tau_{2} / \tau_{1}$ without optimization of the axes is worse than the $\tau_{2} / \tau_{1}$ variants with a "one-pass" optimization. The worst performing variables are the 


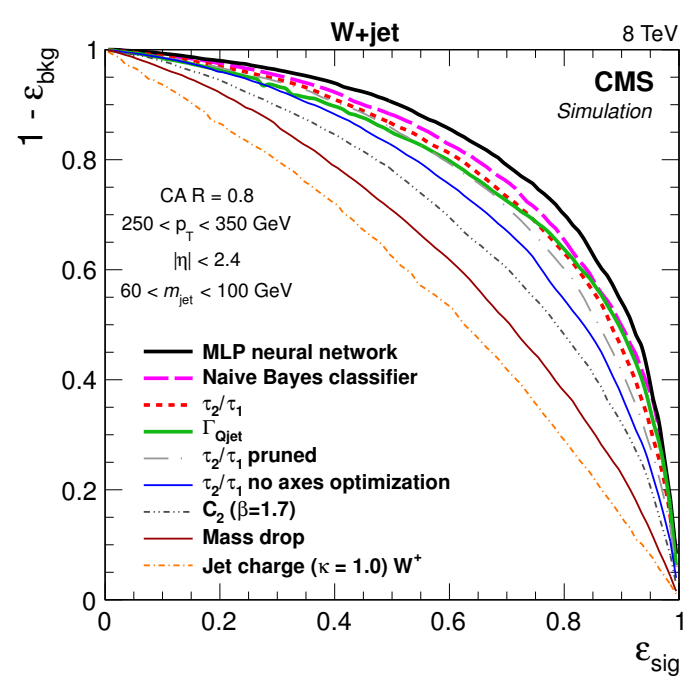

Figure 3. Performance of several discriminants in the background-signal efficiency plane in the low jet $p_{\mathrm{T}}$ bin of $250-350 \mathrm{GeV}$ in the $\mathrm{W}+$ jet topology. The efficiencies and mistagging rates of the various discriminants are estimated on samples of $\mathrm{W}$ jets and QCD jets that satisfy a pruned jet mass selection of $60<m_{\text {jet }}<100 \mathrm{GeV}$.

mass drop, $C_{2}(\beta=1.7)$, and the jet charge. We also find that the discrimination power between $W^{+}$jets and $W^{-}$jets varies by less than $10 \%$ for values of the $\kappa$ parameter in eq. (5.4) between 0.3 and 1.0.

In addition to the performance of individual variables, we study how their combination can improve the separation between $\mathrm{W}$ and QCD jets. A multivariate optimization is performed using the TMVA package [58]. A combination of observables is considered in a naive Bayes classifier and in a Multilayer Perceptron (MLP) neural network discriminant. Additional observables with respect to those shown in figure 3 are used in an attempt to increase the discrimination power. The variables used in both discriminants are the mass drop, $\Gamma_{\text {Qjet }}, \tau_{2} / \tau_{1}, C_{2}^{\beta}$, the jet charge, the planar flow, the number of jet constituents, $\Delta R$ between subjets, sensitivity of trimmed grooming, and the number of primary pp interaction vertices. The MLP neural network is trained using a signal sample from a SM Higgs-like resonance decaying to a pair of longitudinally polarized $\mathrm{W}$ bosons and a background sample of $\mathrm{W}+$ jets generated with MADGRAPH, splitting the events equally in training and test event samples to compute the ROC curve. The ROC curves obtained from the multivariate methods are shown in figure 3. Compared to the performance of $\tau_{2} / \tau_{1}$, a small improvement is obtained using such multivariate discriminators. This can be understood, because we find a large linear correlation between $\tau_{2} / \tau_{1}$, which is the most sensitive variable over a large range of efficiencies, and most of the other observables. We therefore focus in the following of this paper on a baseline tagger based on $\tau_{2} / \tau_{1}$ and point out that, not considering systematic uncertainties, there is potential gain in using multivariate discriminators.

The comparison above is performed after requiring the pruned jet mass to lie in the $\mathrm{W}$ boson mass window. Since all substructure variables are correlated with the jet mass, 

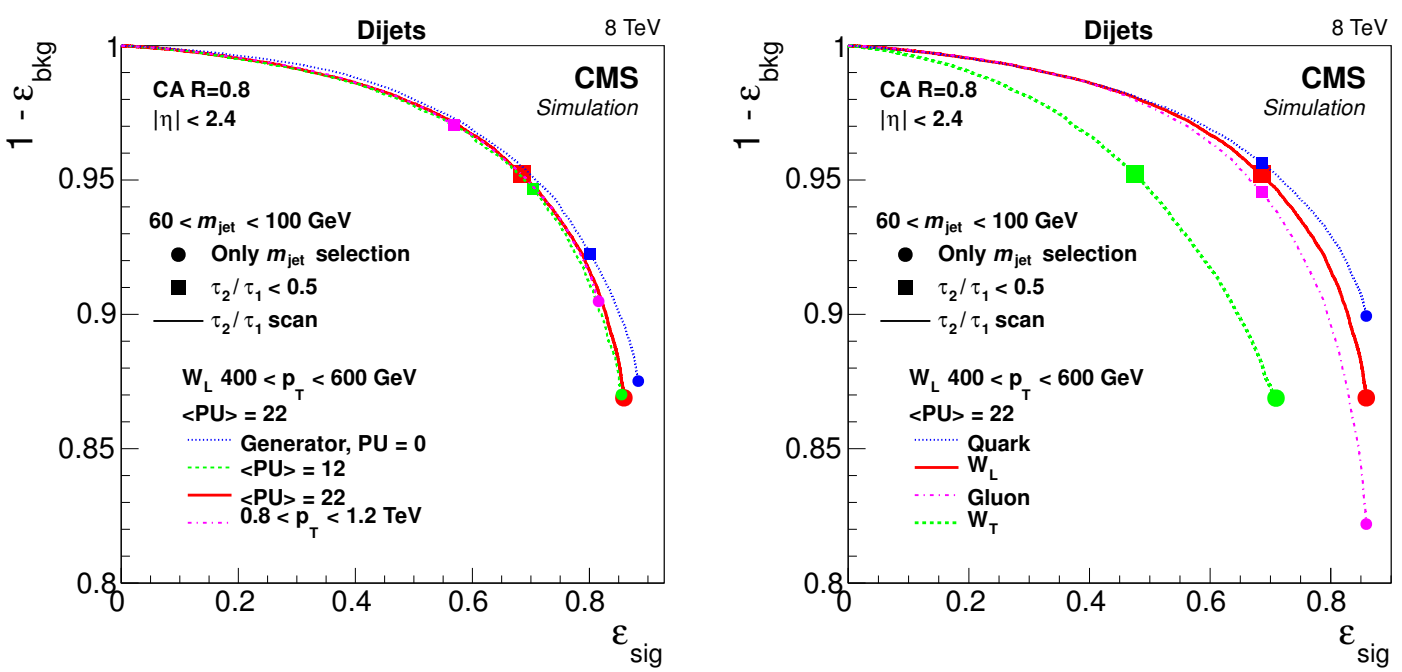

Figure 4. Systematic effects on the performance of the pruned jet mass and $\tau_{2} / \tau_{1}$ W-tagging algorithm in the high jet $p_{\mathrm{T}}$ bin of $400-600 \mathrm{GeV}$. The performance of the pruned jet mass selection $60<m_{\text {jet }}<100 \mathrm{GeV}$ in the various scenarios is indicated as a filled circle. The performance of the combination of $60<m_{\text {jet }}<100 \mathrm{GeV}$ and $\tau_{2} / \tau_{1}<0.5$ is indicated as a filled rectangle. The lines correspond to the ROC curve of a selection on $\tau_{2} / \tau_{1}$ in addition to $60<m_{\text {jet }}<100 \mathrm{GeV}$. The solid line corresponds (in both parts) to the standard scenario with an average of 22 pileup interactions and longitudinally polarized $\mathrm{W}$ bosons $\left(\mathrm{W}_{\mathrm{L}}\right)$.

it is important to note that the variable comparison shown in figure 3 depends strongly on the choice of the primary discriminant. When the ungroomed jet mass is the primary discriminant, a combination with other variables provides a larger increase in discrimination, although the overall performance is still inferior to the default choice of the pruned jet mass and $\tau_{2} / \tau_{1}$.

\subsection{Performance in simulation}

In this section we examine the simulated $p_{\mathrm{T}}$ and $\mathrm{PU}$ dependence of the $\mathrm{W}$ tagging efficiency. Efficiencies are defined for a pruned jet mass of $60<m_{\text {jet }}<100 \mathrm{GeV}$, and N-subjettiness ratio of $\tau_{2} / \tau_{1}<0.5$.

In figure 4, we compare systematic effects in terms of change in the ROC response in the dijet final state for $400<p_{\mathrm{T}}<600 \mathrm{GeV}$. In contrast to figure 3 , where just the performance of other variables was studied relative to that of $m_{\text {jet }}$, here the efficiency is measured for the joint condition on $m_{\text {jet }}$ and $\tau_{2} / \tau_{1}$, demonstrating the impact of these discriminants. The performance for the working point requirements $60<m_{\text {jet }}<100 \mathrm{GeV}$ and $\tau_{2} / \tau_{1}<0.5$ is also indicated. The HERWIG ++ sample is used to model QCD jets, since we observe that it models the pruned jet mass in data better than PYTHIA 6 does. Each of the displayed systematic effects is discussed below.

Figure 5 shows the efficiency of the baseline selection $\left(60<m_{\text {jet }}<100 \mathrm{GeV}\right.$ and $\left.\tau_{2} / \tau_{1}<0.5\right)$ determined from a WW simulation. The efficiency is given as a function of (left) jet $p_{\mathrm{T}}$ and (right) the number of reconstructed vertices, reflecting the contribution from pileup. At low $p_{\mathrm{T}}$, the efficiency increases with $p_{\mathrm{T}}$ for the same reason as in figure 1 , 

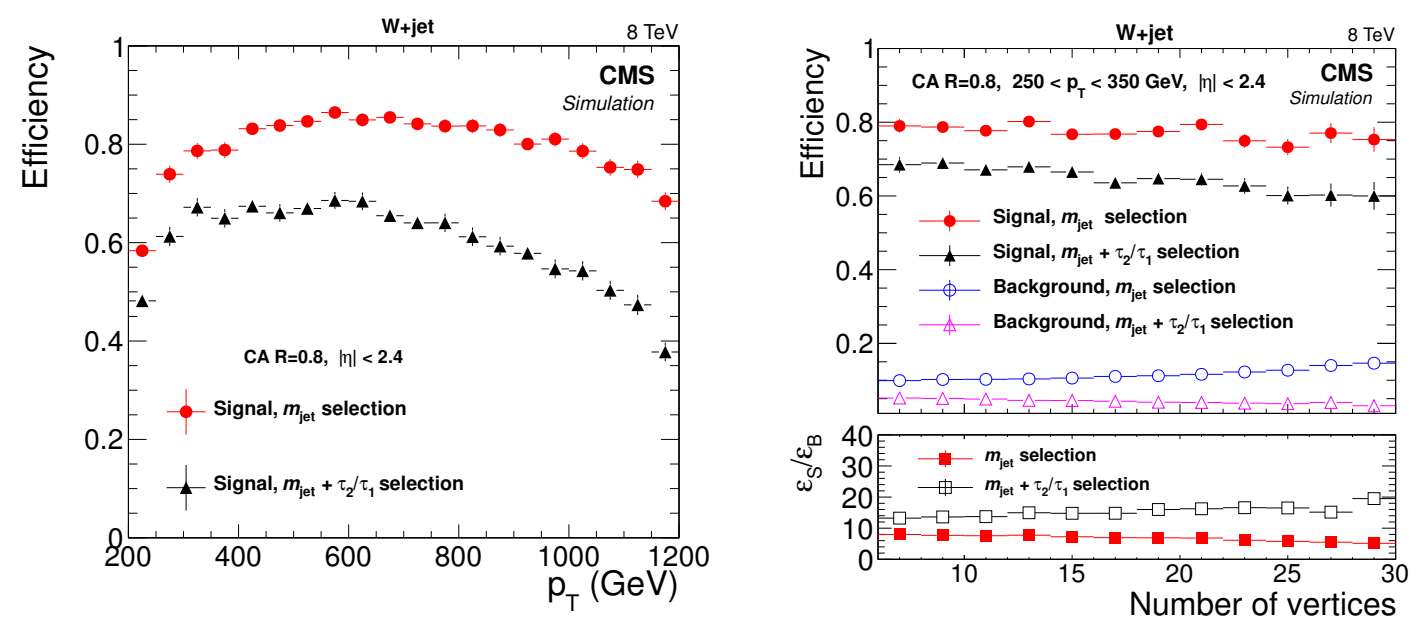

Figure 5. Efficiency of the $m_{\text {jet }}$ selection and the combined $\tau_{2} / \tau_{1}$ and $m_{\text {jet }}$ selection on WW signal samples as a function of (left) $p_{\mathrm{T}}$ and (right) the number of reconstructed vertices. The figure on the right also shows the mistagging rate for QCD jets estimated from the $\mathrm{W}+$ jets background sample. The error bars represent the statistical uncertainty in the MC simulation and the horizontal ones the binning.

namely that at higher $p_{\mathrm{T}}$ the showers from the $\mathrm{W}$ decay quarks are more likely to be reconstructed within a single $\mathrm{CA} 8$ jet. Above $600 \mathrm{GeV}$, the efficiency begins to decrease as a function of jet $p_{\mathrm{T}}$, since at larger $p_{\mathrm{T}}$ the $\mathrm{PF}$ candidate reconstruction degrades in resolving the jet substructure and the pruning algorithm therefore removes too large a fraction of the jet mass. For Run II of the LHC, the particle flow reconstruction has been optimized by making better usage of the segmentation of the ECAL, where we expect to maintain constant efficiency up to at least $p_{\mathrm{T}}=3.5 \mathrm{TeV}$ [59].

The efficiency of the additional $\tau_{2} / \tau_{1}$ selection also drops as a function of $p_{\mathrm{T}}$. It is important to note that the same efficiency at an equivalent background rejection rate can be reached by adjusting the maximum $\tau_{2} / \tau_{1}$ as a function of $p_{\mathrm{T}}$. Figure 4 (left) shows that the ROC curve for jets with $p_{\mathrm{T}}$ between 0.8 and $1.2 \mathrm{TeV}$ (using a $2 \mathrm{TeV}$ mass for the WW resonance) is almost indistinguishable from the ROC curve derived from the $400-$ $600 \mathrm{GeV} p_{\mathrm{T}}$ range, except that the working point corresponding to $\tau_{2} / \tau_{1}<0.5$ (square) is at a lower signal efficiency. Consequently, a fixed working point will degrade the efficiency with increasing $p_{\mathrm{T}}$. However, by shifting the working point, the same performance can be achieved.

The efficiency of the $m_{\text {jet }}$ selection as a function of the number of reconstructed vertices, shown in figure 5 (right), decreases by $6 \%$ between 5 and 30 reconstructed vertices, whereas the additional $\tau_{2} / \tau_{1}$ selection efficiency drops by $12 \%$ over the same range. However, the mistagging of the background also decreases with pileup for the same selection, yielding similar discrimination. Efficiency and mistagging rate are affected by pileup in the same way, since additional pileup shifts the $\tau_{2} / \tau_{1}$ distribution towards higher values (towards background like) for both signal and background. Therefore, the same signal efficiency can be reached at the same background rejection rate for up to 30 reconstructed vertices by merely adjusting the $\tau_{2} / \tau_{1}$ selection, as demonstrated in figure 4 (left). Moving from an average pileup of 12 to 22 interactions shows almost no change in the ROC response. 
We also study the performance of jet substructure tagging algorithms by convolving pileup, CMS detector resolution, and efficiencies in reconstructing the particles that form the jets. In figure 4 (left), the generator level predictions without pileup are compared with the performance after full CMS simulation with pileup. A small degradation is observed relative to generator level, but the performance at detector level is almost as good as predicted at particle level, although the $\mathrm{W}$ jet and the QCD jet $\tau_{2} / \tau_{1}$ distributions are shifted up significantly by pileup and detector effects, as seen in figure 2 .

\subsection{W-polarization and quark-gluon composition}

An important factor that influences the $\mathrm{W}$-tagging performance is the polarization of the reconstructed $\mathrm{W}$ bosons. Furthermore, the $\mathrm{W}$ polarization can be used to identify the nature of any new phenomena, such as, for example, through studies of new WW resonances, $\mathrm{W}$ boson helicities at large $t \overline{\mathrm{t}}$ masses, or WW scattering. We study the effect of $\mathrm{W}$ polarization by comparing simulated samples of $X \rightarrow \mathrm{WW}$, where the $\mathrm{W}$ bosons are either purely longitudinally $\left(\mathrm{W}_{\mathrm{L}}\right)$ or transversely $\left(\mathrm{W}_{\mathrm{T}}\right)$ polarized. The key observable is the helicity angle of $\mathrm{W} \rightarrow \overline{\mathrm{q}} \mathrm{q}^{\prime}$ decays $\left(\cos \theta_{J}\right)$ as defined in the rest frame of the $\mathrm{W}$ boson relative to the $\mathrm{W}$ direction of motion [31]. The distribution of $\cos \theta_{J}$ at the parton level, where quarks are treated as final state particles, is presented in figure 6 (left). After reconstruction, the polarization in $\mathrm{W}$ jets can be recovered using the pruned subjets as a proxy for the $\mathrm{W}$ decay quarks. However, using the subjets, it is not possible to distinguish the fermion and antifermion in the $\mathrm{W}$ decay, which restricts the distributions to $0 \leq \cos \theta_{J} \leq 1$. Figure 6 (right) shows the helicity angle between the two pruned subjets for a $600 \mathrm{GeV} \mathrm{X}$ resonance, differing from figure 6 (left) in that it includes reconstruction and acceptance effects. The depletion of events at $\left|\cos \theta_{J}\right| \approx 1$ is due to two acceptance effects. When $\theta_{J} \approx 0$, the partons would be overlapping and thus reconstruction of two subjets is difficult. When $\theta_{J} \approx \pi$, the one subjet tends to be much softer than the other and this can cause the loss or misidentification of the subjet originating from one of the $\mathrm{W}$ decay partons. It appears that transversely polarized $\mathrm{W}$ bosons decay with the quarks emitted closer to the direction of the $\mathrm{W}$, and therefore can be used to determine the polarization of the $\mathrm{W}$ boson. Going further, the reconstructed $\cos \theta_{J}$ is compared to the parton-level information. The resolution on the angular distance between two subjets in the laboratory frame is approximately $10 \mathrm{mrad}$, which translates to a resolution of approximately $65 \mathrm{mrad}$ on $\theta_{J}$ in the $\mathrm{W}$ rest frame. The resolution remains relatively constant over a large range of $\mathrm{W}$ jet $p_{\mathrm{T}}$.

Figure 4 (right) compares the signal-to-background discrimination of the $\mathrm{W}$ tagger for pure $\mathrm{W}_{\mathrm{L}}$ and pure $\mathrm{W}_{\mathrm{T}}$ signal samples. We observe that the pruned jet mass selection is less efficient for $\mathrm{W}_{\mathrm{T}}$; this is consistent with figure 6 (right), where the $\mathrm{W}_{\mathrm{T}}$ jets with $\left|\cos \theta_{J}\right| \approx 1$ are removed by the pruned jet mass selection. This can be explained by a higher asymmetry in the $p_{\mathrm{T}}$ of the two quarks from the $\mathrm{W}_{\mathrm{T}}$ decay, such that the pruning algorithm in a considerable fraction of events rejects the particles from the lower $p_{\mathrm{T}}$ quark and yields a much lower jet mass. In addition, the $\Delta R$ separation between the partons for pure $\mathrm{W}_{\mathrm{L}}$ bosons is smaller on average than for $\mathrm{W}_{\mathrm{T}}$ bosons and is more likely to be accepted by a CA 8 jet. Of the two effects, the dominant contribution depends on the 

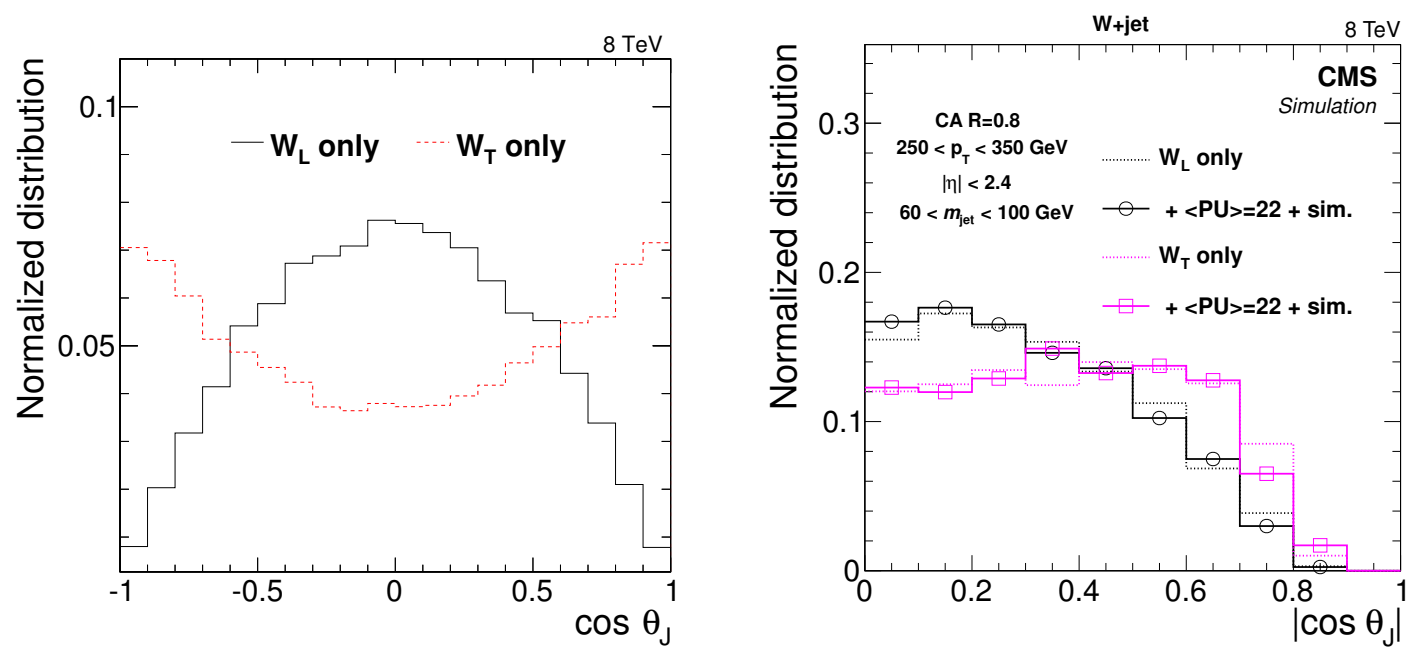

Figure 6. Left: generator level $\cos \theta_{J}$ distributions for longitudinally and transversely polarized $\mathrm{W}$ bosons. Right: subjet angular observables after a selection on pruned jet mass of $\mathrm{W}_{\mathrm{L}}$ and $\mathrm{W}_{\mathrm{T}}$ samples for jets with $250<p_{\mathrm{T}}<350 \mathrm{GeV}$.

transverse momentum of the $\mathrm{W}$ jet. For higher jet $p_{\mathrm{T}}$, the difference in the reconstructed $\cos \theta_{J}$ and $\Delta R$ between $\mathrm{W}_{\mathrm{L}}$ and $\mathrm{W}_{\mathrm{T}}$ becomes larger since the more QCD-like topology of the transversely polarized $\mathrm{W}$ bosons becomes important, i.e. it is easier to distinguish $\mathrm{W}_{\mathrm{L}}$ and $\mathrm{W}_{\mathrm{T}}$. The $\tau_{2} / \tau_{1}$ discrimination power is also degraded for $\mathrm{W}_{\mathrm{T}}$, although, to a smaller degree than the pruned jet mass.

The composition of the QCD background also influences the discrimination of the variables discussed in section 5 , since the properties of quark- and gluon-initiated jets differ. For example, gluon jets tend to have a larger jet mass than quark jets and therefore fewer gluon jets are rejected by the pruned jet mass selection; this can be seen in figure 4 (right). On the contrary, the $\tau_{2} / \tau_{1}$ discriminator rejects more gluon jets than quark jets and for these reasons a similar performance for quarks and gluons is achieved for the working point of $\tau_{2} / \tau_{1}<0.5$.

\section{Performance in data and systematic uncertainties}

\subsection{Comparison of data and simulation}

We compare the distributions of substructure observables between simulation and data in inclusive dijet, $\mathrm{W}+$ jet and $\mathrm{t} \overline{\mathrm{t}}$ samples. The $\mathrm{W}+$ jet and dijet events are compared in

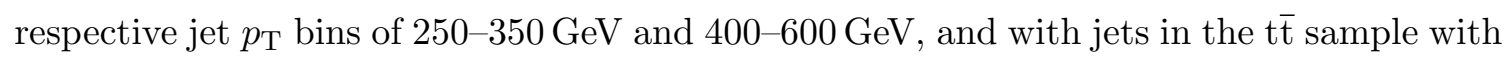
$p_{\mathrm{T}}>200 \mathrm{GeV}$. Simulation with different parton shower models of PYтніA 6 , PYTHIA 8 and HERWIG ++ are also compared.

In figure 7, the pruned jet mass distribution is shown for both data and simulation in the dijet and $\mathrm{W}+$ jet samples that probe the $\mathrm{W}$-tagging variables using $\mathrm{QCD}$ jets. We find that the agreement is good between data and simulation, but HERWIG ++ agrees better than PYTHia 6 , and PYтнia 8 shows best agreement. Similar findings have been reported 

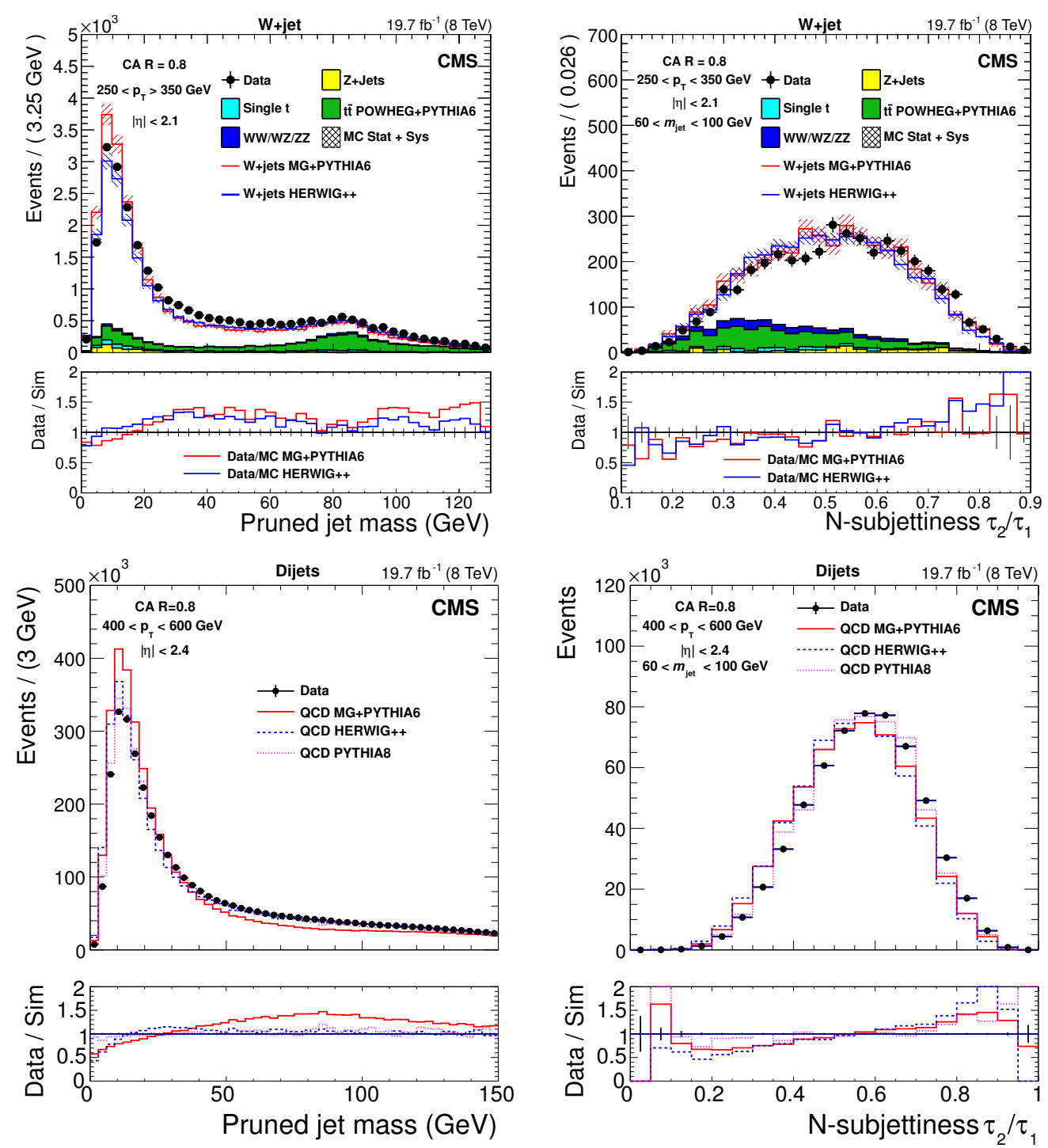

Figure 7. Pruned jet mass and $\mathrm{N}$-subjettiness ratio $\tau_{2} / \tau_{1}$ distributions in data and simulation for $\mathrm{W}+$ jets events in (upper left) and (upper right) and for dijet events in (lower left) and (lower right). MG denotes the MADGRAPH generator. Below each figure the relative deviations are plotted between data and simulations.

in refs. $[1,2,4]$. The $\tau_{2} / \tau_{1}$ variable is also shown and found to agree better with HERWIG++ and best with PYTHIA 8 .

To probe the description of $\mathrm{W}$ jets, we use the control sample of pure $\mathrm{W}$ bosons in the data from the high $p_{\mathrm{T}}$ lepton+jets t $\overline{\mathrm{t}}$ sample. The pruned jet mass and $\tau_{2} / \tau_{1}$ distributions in the $t \bar{t}$ control sample are shown in figure 8 for the muon selection. The plots include systematic and statistical uncertainties, where the band of systematic uncertainty represents the normalization uncertainties on the VV, single top quark and $\mathrm{W}+$ jets cross sections. The systematic uncertainty is estimated to be $20 \%$ determined from the relative difference in the mean value between the recent cross section measurement at $\sqrt{s}=8 \mathrm{TeV}$ at CMS and the SM expectation [60]. The agreement between simulation and data is 

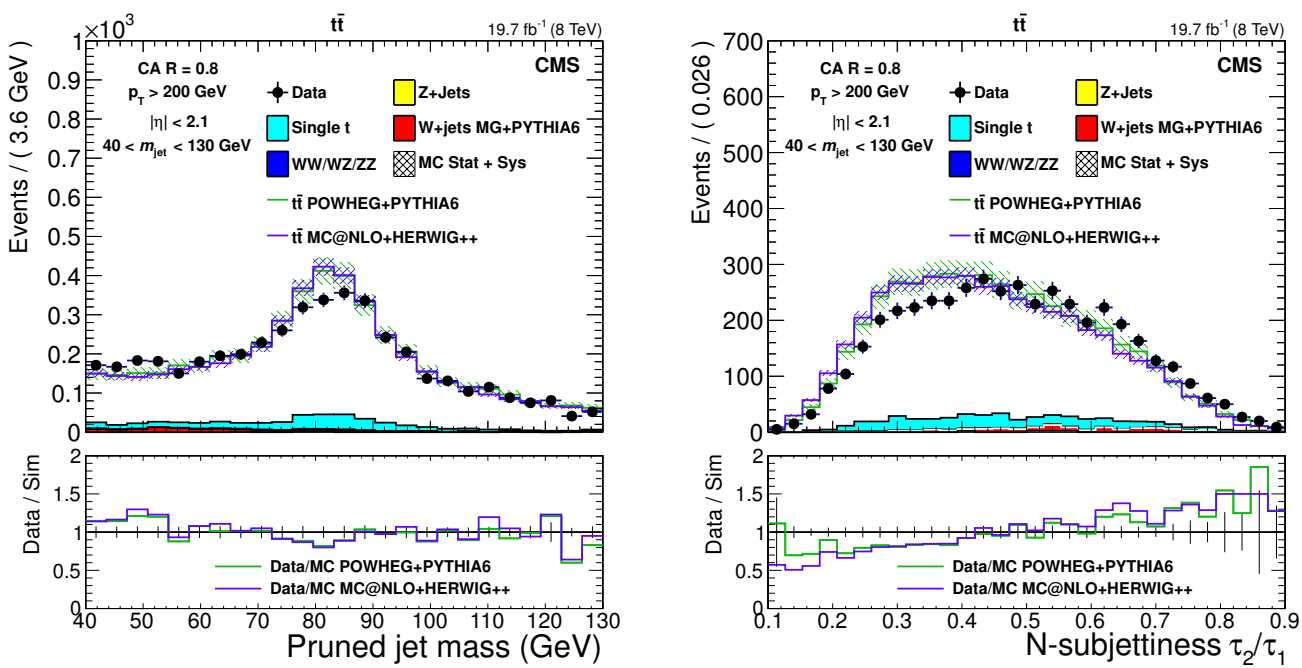

Figure 8. Pruned jet mass and $\tau_{2} / \tau_{1}$ distributions for the lepton+jets $t \overline{\mathrm{t}}$ control sample for the muon selection. Below each figure the relative deviations are plotted between data and simulations.

reasonable, but there are discrepancies of the order of $10 \%$. In section 6.3 we describe the derivation of data-to-simulation scale factors to correct for these discrepancies. Generally,

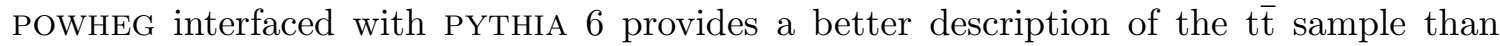
MC@NLO interfaced with HERWIG++.

Finally, we compare the jet charge distribution of $\mathrm{W}$ jets in data and in simulation using the t $\bar{t}$ sample. By selecting a negatively or positively charged lepton, we can effectively choose a $\mathrm{W}^{+}$or $\mathrm{W}^{-}$jet. This can be seen in figure 9 . While $\mathrm{W}^{+}$and $\mathrm{W}^{-}$jets can't be distinguished on an event-by-event basis, their contributions to the t $\overline{\mathrm{t}}$ data sample can be separated with a significance larger than 5 standard deviations. The jet charge distribution is well described by the simulation.

\subsection{Mistagging rate measurement}

A dijet sample is used to measure the rate of false positive $\mathrm{W}$ tags, or mistags. The mistagging rate is measured in data and compared to simulation. As discussed previously, the $\mathrm{W}$ tagger selection requires $60<m_{\text {jet }}<100 \mathrm{GeV}$ and $\tau_{2} / \tau_{1}<0.5$. Figure 10 shows the fraction of jets passing just the $m_{\text {jet }}$ requirement, as well as the simultaneous $m_{\text {jet }}$ and $\tau_{2} / \tau_{1}$ requirements, as a function of $p_{\mathrm{T}}$ and of the number of reconstructed vertices. Similarly as in the case of the efficiency, the mistagging rate for the $m_{\text {jet }}$ and $\tau_{2} / \tau_{1}$ selections decreases as a function of $p_{\mathrm{T}}$. The mistagging rate of only the $m_{\text {jet }}$ requirement in data is well reproduced by HERWIG ++ and PYTHIA 8, while MADGRAPH+PYTHIA 6 underestimates it. When both the $m_{\text {jet }}$ and $\tau_{2} / \tau_{1}$ requirements are applied, the mistagging rate in data is reproduced better by PYTHIA 8 than by MADGRAPH+PYTHIA 6 and HERWIG ++ . The $p_{\mathrm{T}}$ dependence in data is well reproduced by all generators.

As a function of pileup, the mistagging rate is stable within $1 \%$ for the $m_{\text {jet }}$ selection. The mistagging rate for the combination of the $m_{\text {jet }}$ and $\tau_{2} / \tau_{1}$ selections drops as a function 


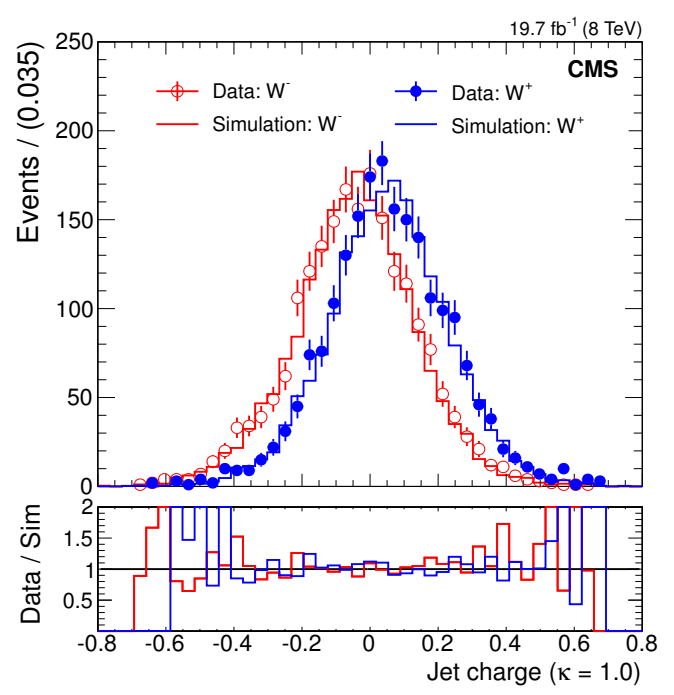

Figure 9. Jet charge distributions in the $t \bar{t}$ control sample in simulation and data for $\mathrm{W}^{+}$and $\mathrm{W}^{-}$jets selected by requiring negatively and positively charge leptons, respectively. Simulated

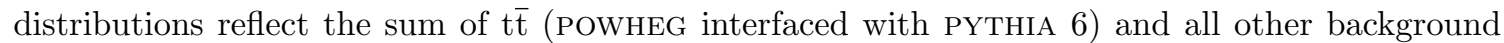
processes. Below each figure the relative deviations are plotted between data and simulations.
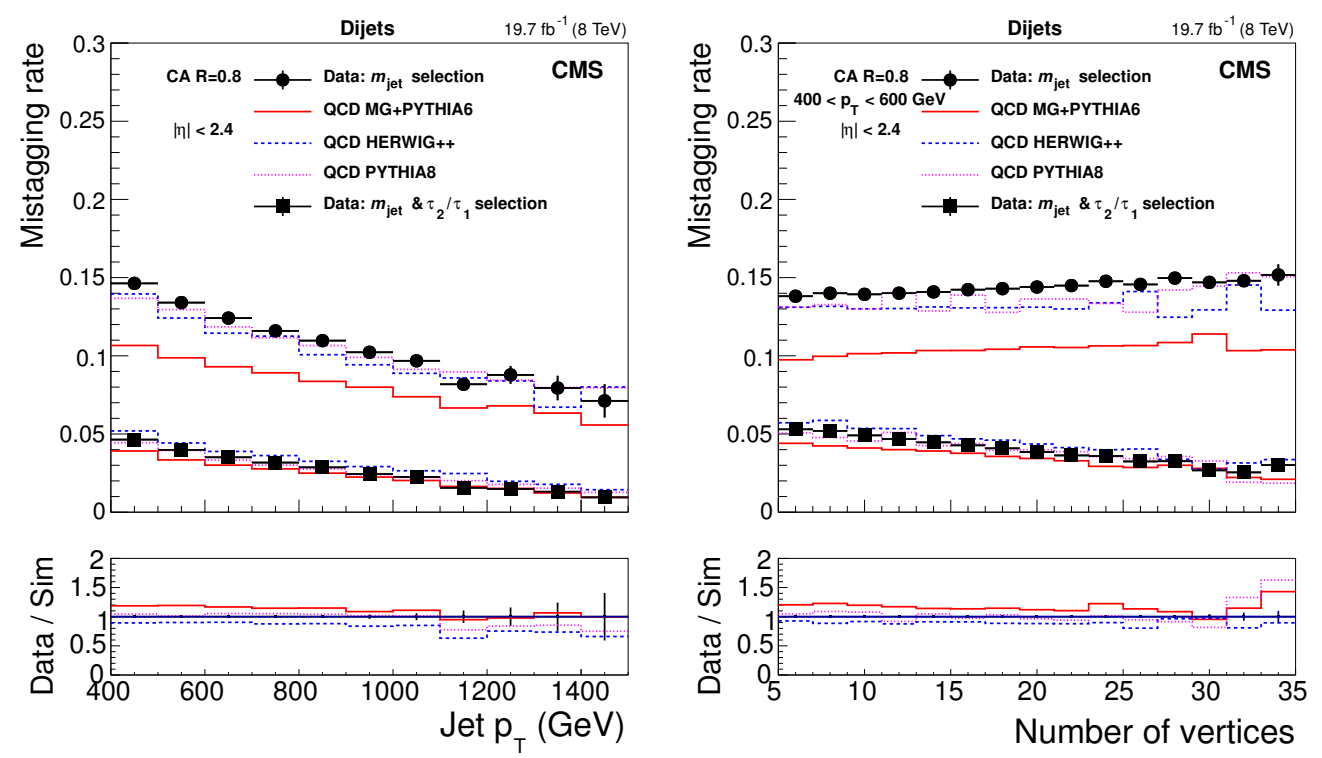

Figure 10. Fraction of jets passing the $m_{\text {jet }}$ and $\tau_{2} / \tau_{1}$ selections in dijet data sample and simulation as a function of (left) $p_{\mathrm{T}}$ and (right) the number of reconstructed vertices. The data over simulation ratio is shown for the combination of the $m_{\text {jet }}$ and $\tau_{2} / \tau_{1}$ selections.

of pileup as discussed in detail in section 5.3. The PU dependence is well reproduced by the simulation.

\subsection{Efficiency scale factors and mass scale/resolution measurement}

The $t \bar{t}$ control sample is used to extract data-to-simulation scale factors for the $\mathrm{W}$ jet efficiency. These factors are meant to correct the description of the $\mathrm{W}$-tagging efficiency in 
the simulation. They depend on the definition of the $\mathrm{W}$-tagger as well as the $\mathrm{MC}$ generator used for simulation. We demonstrate the extraction of data-to-simulation scale factors for a simple selection on $\tau_{2} / \tau_{1}<0.5$, and jet mass scale, and resolution based on a simulation using POWHEG interfaced with PYTHIA 6 . We are concerned only with the efficiency for the pure $\mathrm{W}$ jet signal, and must therefore subtract background contributions to measure the scale factors. The pruned jet mass distribution is used to discriminate the pure $\mathrm{W}$

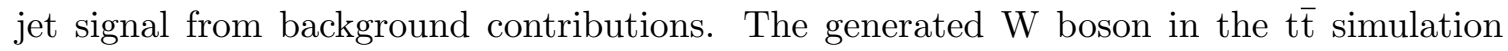
provides a model of the contribution from the $\mathrm{W}$ jet peak in the pruned jet mass. The contribution from combinatorial background is derived from t $\bar{t}$ simulation as well. This model is fitted directly in the distributions of data and in their simulation.

The scale factors $(\mathrm{SF})$ for the selection on $\tau_{2} / \tau_{1}<0.5$ are extracted by estimating the selection efficiency on both data and simulation. The pruned jet mass distribution of events that pass and fail the $\tau_{2} / \tau_{1}$ selection are fitted simultaneously to extract the selection efficiency on the pure $\mathrm{W}$ jet component as shown in figure 11. The ratio of data and simulation efficiencies are taken as the $\mathrm{W}$-tagging efficiency SF. In the t $\overline{\mathrm{t}}$ control region we use a mass window of $65-105 \mathrm{GeV}$, because of a slight shift in the mean mass of the $\mathrm{W}$ boson peak in $\mathrm{t} \overline{\mathrm{t}}$ events of $\approx 1.5 \mathrm{GeV}$. In simulation the slight shift in mass is found to be primarily due to extra radiation in the $\mathrm{W}$ jet from the nearby $\mathrm{b}$ quark. Additional

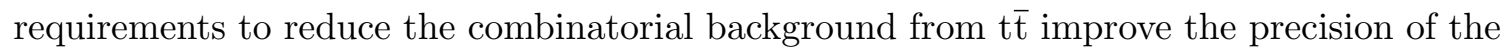
determined scale factor. Therefore, the angular distance $\Delta R$ between the $\mathrm{W}$ jet candidate and the closest b-tagged AK5 jet is required to be less than 2.0, which is typical for highly boosted top quark decays [2]. This additional selection reduces the uncertainty on the scale factor by $21 \%$. Further reduction of the combinatorial background can be achieved through

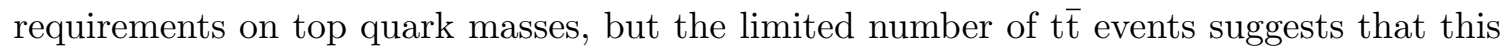
can become relevant only with a larger data sample. The results of the fit are shown in figure 11. We find the "pass" sample agrees well between the data and simulation while the "fail" sample is not as well modeled, particularly when the failing jet is not a fully merged $\mathrm{W}$ boson but a quark or gluon jet. This is compensated in our computation of the data-to-MC scale factor. The scale factor is computed to be $0.93 \pm 0.06$. The uncertainty in the SF is purely statistical. In section 6.4 , we discuss systematic effects to this scale factor. The $p_{\mathrm{T}}$ dependence of the scale factor was also studied at a limited statistical precision. In two $p_{\mathrm{T}}$ bins between $200-265$ and $265-600 \mathrm{GeV}$ the scale factors were found to be $1.00 \pm 0.09$ and $0.92 \pm 0.10$, respectively. No significant $p_{\mathrm{T}}$ dependence of the scale factor is observed.

To extract corrections to the jet mass scale and resolution, we use the mean $\langle m\rangle$ and resolution $\sigma$ value of the Gaussian component of the fitted function of the $\mathrm{W}$ bosons in the passed sample. Since we do not expect the jet mass scale and resolution to differ between electron and muon channels, the muon and electron data are fitted simultaneously, forcing the $\langle m\rangle$ and $\sigma$ of the Gaussian component of the fit to be the same in the two channels. The fits are shown for the $\tau_{2} / \tau_{1}<0.5$ selection in figure 11 (left column), and the resulting parameters are summarized in table 1 . We find that both the $\mathrm{W}$ jet mass scale and resolution in data are larger than that in simulation. In the simulation $\langle m\rangle$ must therefore be shifted by $1.7 \pm 0.6 \%$ and $\sigma$ be enlarged by $11 \pm 9 \%$ to correct for the difference between data and simulation. 

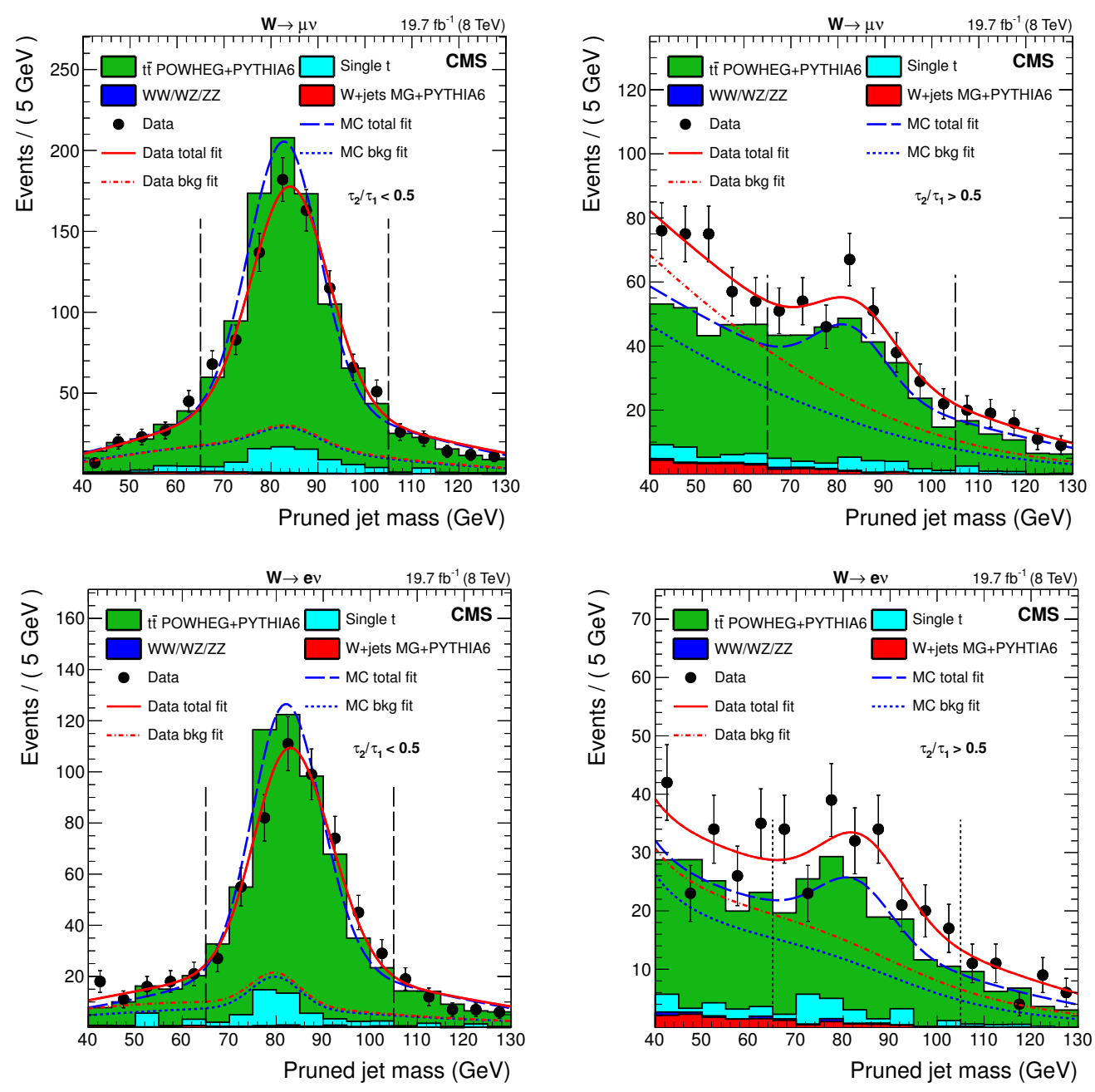

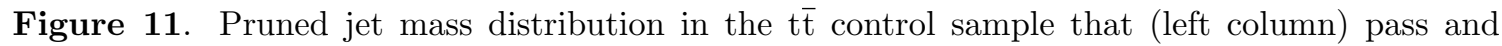
(right column) fail the $\tau_{2} / \tau_{1}<0.5$ selection for the (upper row) muon, and for the (lower row) electron channels. The result of the fit to data and simulation are shown, respectively, by the solid and long-dashed line and the background components of the fit are shown as dashed-dotted and short-dashed line.

\begin{tabular}{|c|ccc|}
\hline Parameter & Data & Simulation & Data/Simulation \\
\hline$\langle m\rangle$ & $84.1 \pm 0.4 \mathrm{GeV}$ & $82.7 \pm 0.3 \mathrm{GeV}$ & $1.017 \pm 0.006$ \\
$\sigma$ & $8.4 \pm 0.6 \mathrm{GeV}$ & $7.6 \pm 0.4 \mathrm{GeV}$ & $1.11 \pm 0.09$ \\
\hline
\end{tabular}

Table 1. Summary of the fitted W-mass peak fit parameters.

\subsection{Systematic uncertainties}

We now discuss systematic uncertainties in the $\mathrm{W}$ tagging scale factor. Several important effects, including the modeling of parton shower and the PDF, polarization of the $\mathrm{W}$ boson, the pileup, presence of nearby jets, the jet mass scale, jet energy scale, and resolution effects, as well as less dominant contributions from the uncertainties in lepton identification, 
b tagging and $E_{\mathrm{T}}^{\text {miss }}$ scale are considered. The effects from the modeling of the parton shower and the PDF are quantified by the difference between the efficiency in a t $\overline{\mathrm{t}}$ sample generated with POWHEG interfaced with PYTHIA 6 and a sample from MC@NLO interfaced with HERWIG++. The effects from modeling the underlying event in the simulation are estimated by comparing three alternative tunes (Z2*, AMBT1 [61] and AMBT2 [62]) of the multiple parton interaction model in the PYтнIA 6 simulation, and taking the maximal observed difference as an estimate of the uncertainty.

As we have shown above, the polarization of the $\mathrm{W}$ boson has a significant impact on the W-tagging efficiency, which has to be taken into account when propagating the scale factor from $t \bar{t}$ events to other final states. The $\mathrm{W}$ boson polarization in $t \bar{t}$ events has been measured by CMS with a precision of 4\% [63]. Although the agreement in this result between data and theory was found to be less than the quoted precision, we nevertheless use this number as an upper limit and include it into an uncertainty of the $\mathrm{W}$ tagging scale factor.

The effect from jet mass scale and resolution is evaluated by changing them by the uncertainty in the fitted mean and resolution, estimated in section 6.3. Their impact on the $\mathrm{W}$-tagging efficiency is small, since the $\mathrm{W}$ boson mass peak is well within the chosen pruned jet mass window.

The jet energy scale and resolution are changed within their $p_{\mathrm{T}^{-}}$and $\eta$-dependent uncertainties [23,64]. The impact of nearby jets on the scale factor is estimated by comparing it to a scale factor for an explicit requirement on the angular distance between the closest AK5 jet and the $\mathrm{W}$ jet of $\Delta R>1.3$. The uncertainty from pileup is determined by moving the minimum bias cross section within its measured uncertainty of $6 \%$ [65]. The scale factors for lepton and b jet identification are also changed within their uncertainties. Finally, uncertainties in the energy and momentum scale and resolution of leptons and jets in the event are propagated to an uncertainty on the $E_{\mathrm{T}}^{\text {miss }}$.

The results are summarized in table 2. The dominant systematic effect on the scale factor for the efficiency is from modeling of the parton shower and PDF, with a systematic uncertainty of $6.0 \%$. The quadratic sum of systematic uncertainties of $7.6 \%$ is comparable to the statistical uncertainty on the scale factor of $6.4 \%$.

\section{Summary and outlook}

In this paper we presented techniques for the identification of jets originating from highly boosted $\mathrm{W}$ bosons that decay into $\overline{\mathrm{q}} \mathrm{q}^{\prime}$, where the final decay products are reconstructed within a single jet, called a $\mathrm{W}$ jet. The pruned jet mass, used as the primary identifying observable for $\mathrm{W}$ jets, and several substructure observables that can provide additional signal to background discrimination, were evaluated for their impact.

The investigated substructure observables were three variants of the $\mathrm{N}$-subjettiness $\tau_{2} / \tau_{1}$, the mass drop, the Qjet volatility, the double ratio of the energy-correlation function $C_{2}^{\beta}$, and the jet charge. Effects from pileup, detector resolution, polarization of the $\mathrm{W}$ boson, and the quark/gluon composition of QCD jets, as well as the performance of the discriminant at large $p_{\mathrm{T}}$ were studied. The results were evaluated after applying a pruned 


\begin{tabular}{|c|c|}
\hline Source & Effect on the scale factor \\
\hline Parton showering + PDF & $6.0 \%$ \\
Underlying event & $<0.5 \%$ \\
W-polarization & $2.0 \%$ \\
Pileup & $1.8 \%$ \\
Nearby jets & $2.4 \%$ \\
Jet mass scale & $<0.5 \%$ \\
Jet mass resolution & $1.9 \%$ \\
Jet energy scale & $1.9 \%$ \\
Jet energy resolution & $0.9 \%$ \\
Lepton ID & $<0.5 \%$ \\
b-tagging & $<0.5 \%$ \\
$E_{\mathrm{T}}^{\text {miss }}$ & $<0.5 \%$ \\
\hline Total systematic & $7.6 \%$ \\
\hline Statistical & $6.4 \%$ \\
\hline Total & $9.9 \%$ \\
\hline
\end{tabular}

Table 2. Summary of uncertainties on the $\mathrm{W}$ jet identification efficiency scale factor.

jet mass selection, and the $\tau_{2} / \tau_{1}$ with one-pass optimized $k_{\mathrm{T}}$ axes was found to be the single most discriminating observable over a large range of signal efficiency. Combining all observables into a multivariate discriminant, indicated a small improvement relative to just $\tau_{2} / \tau_{1}$.

The observables were compared in data and in the simulations, in both dijet and $\mathrm{W}+$ jet topologies. Selecting these topologies provided complementary samples for the jet $p_{\mathrm{T}}$ range, and for the background composition of light-quark- and gluon-initiated jets. Reasonable agreement was found. In general, the HERWIG ++ and PYTHIA 8 generators provide better modeling of jet substructure observables than PYтніA 6. A lepton+jets t $\overline{\mathrm{t}}$ sample was used to select $\mathrm{W}$ jets in data, and this was compared to simulation. In this sample, we also demonstrated discrimination of the jet charge observable in data with $\mathrm{W}^{+}$jets and $\mathrm{W}^{-}$ jets, and we studied the performance of the $\mathrm{W}$-jet tagging algorithm for a specific set of selections. The efficiency and mistagging rate were obtained as a function of $p_{\mathrm{T}}$. For a typical working point, an efficiency of $65 \%$ and a background rejection of $96 \%$ is achieved at $p_{\mathrm{T}}=500 \mathrm{GeV}$. The mistagging rate for a broad range of $p_{\mathrm{T}}$ agrees reasonably with

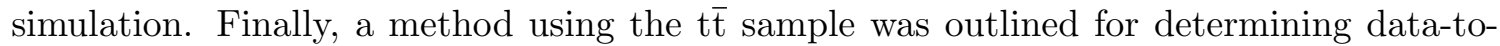
simulation scale factors for correcting differences between data and simulation of the $\tau_{2} / \tau_{1}$ selection, the mass scales, and the resolution.

The methods introduced in this paper are directly applicable for identifying other 
massive objects that decay to hadrons. For identifying $\mathrm{Z}$ jets, the only difference is that the jet mass window is slightly higher. For identifying highly boosted Higgs bosons decaying to bottom quarks, the performance of these observables should be similar. An additional discriminating variable for Higgs that is not addressed in this study, is the possible tagging of b-jets. We leave to future studies the optimization of the method for the boosted Higgs bosons.

\section{Acknowledgments}

We congratulate our colleagues in the CERN accelerator departments for the excellent performance of the LHC and thank the technical and administrative staffs at CERN and at other CMS institutes for their contributions to the success of the CMS effort. In addition, we gratefully acknowledge the computing centres and personnel of the Worldwide LHC Computing Grid for delivering so effectively the computing infrastructure essential to our analyses. Finally, we acknowledge the enduring support for the construction and operation of the LHC and the CMS detector provided by the following funding agencies: the Austrian Federal Ministry of Science, Research and Economy and the Austrian Science Fund; the Belgian Fonds de la Recherche Scientifique, and Fonds voor Wetenschappelijk Onderzoek; the Brazilian Funding Agencies (CNPq, CAPES, FAPERJ, and FAPESP); the Bulgarian Ministry of Education and Science; CERN; the Chinese Academy of Sciences, Ministry of Science and Technology, and National Natural Science Foundation of China; the Colombian Funding Agency (COLCIENCIAS); the Croatian Ministry of Science, Education and Sport, and the Croatian Science Foundation; the Research Promotion Foundation, Cyprus; the Ministry of Education and Research, Estonian Research Council via IUT23-4 and IUT236 and European Regional Development Fund, Estonia; the Academy of Finland, Finnish Ministry of Education and Culture, and Helsinki Institute of Physics; the Institut National de Physique Nucléaire et de Physique des Particules / CNRS, and Commissariat à l'Énergie Atomique et aux Énergies Alternatives / CEA, France; the Bundesministerium für Bildung und Forschung, Deutsche Forschungsgemeinschaft, and Helmholtz-Gemeinschaft Deutscher Forschungszentren, Germany; the General Secretariat for Research and Technology, Greece; the National Scientific Research Foundation, and National Innovation Office, Hungary; the Department of Atomic Energy and the Department of Science and Technology, India; the Institute for Studies in Theoretical Physics and Mathematics, Iran; the Science Foundation, Ireland; the Istituto Nazionale di Fisica Nucleare, Italy; the Korean Ministry of Education, Science and Technology and the World Class University program of NRF, Republic of Korea; the Lithuanian Academy of Sciences; the Ministry of Education, and University of Malaya (Malaysia); the Mexican Funding Agencies (CINVESTAV, CONACYT, SEP, and UASLP-FAI); the Ministry of Business, Innovation and Employment, New Zealand; the Pakistan Atomic Energy Commission; the Ministry of Science and Higher Education and the National Science Centre, Poland; the Fundação para a Ciência e a Tecnologia, Portugal; JINR, Dubna; the Ministry of Education and Science of the Russian Federation, the Federal Agency of Atomic Energy of the Russian Federation, Russian Academy of Sciences, and the Russian Foundation for Basic Research; the Ministry of Education, Sci- 
ence and Technological Development of Serbia; the Secretaría de Estado de Investigación, Desarrollo e Innovación and Programa Consolider-Ingenio 2010, Spain; the Swiss Funding Agencies (ETH Board, ETH Zurich, PSI, SNF, UniZH, Canton Zurich, and SER); the Ministry of Science and Technology, Taipei; the Thailand Center of Excellence in Physics, the Institute for the Promotion of Teaching Science and Technology of Thailand, Special Task Force for Activating Research and the National Science and Technology Development Agency of Thailand; the Scientific and Technical Research Council of Turkey, and Turkish Atomic Energy Authority; the National Academy of Sciences of Ukraine, and State Fund for Fundamental Researches, Ukraine; the Science and Technology Facilities Council, U.K.; the US Department of Energy, and the US National Science Foundation.

Individuals have received support from the Marie-Curie programme and the European Research Council and EPLANET (European Union); the Leventis Foundation; the A. P. Sloan Foundation; the Alexander von Humboldt Foundation; the Belgian Federal Science Policy Office; the Fonds pour la Formation à la Recherche dans l'Industrie et dans l'Agriculture (FRIA-Belgium); the Agentschap voor Innovatie door Wetenschap en Technologie (IWT-Belgium); the Ministry of Education, Youth and Sports (MEYS) of the Czech Republic; the Council of Science and Industrial Research, India; the HOMING PLUS programme of Foundation for Polish Science, cofinanced from European Union, Regional Development Fund; the Compagnia di San Paolo (Torino); the Consorzio per la Fisica (Trieste); MIUR project 20108T4XTM (Italy); the Thalis and Aristeia programmes cofinanced by EU-ESF and the Greek NSRF; and the National Priorities Research Program by Qatar National Research Fund.

Open Access. This article is distributed under the terms of the Creative Commons Attribution License (CC-BY 4.0), which permits any use, distribution and reproduction in any medium, provided the original author(s) and source are credited.

\section{References}

[1] CMS collaboration, Studies of jet mass in dijet and $W / Z+$ jet events, JHEP 05 (2013) 090 [arXiv:1303.4811] [INSPIRE].

[2] CMS collaboration, Jet substructure algorithms, CMS-PAS-JME-10-013 (2010).

[3] ATLAS collaboration, Jet mass and substructure of inclusive jets in $\sqrt{s}=7 \mathrm{TeV} p p$ collisions with the ATLAS experiment, JHEP 05 (2012) 128 [arXiv:1203.4606] [INSPIRE].

[4] ATLAS collaboration, Performance of jet substructure techniques for large-R jets in proton-proton collisions at $\sqrt{s}=7 \mathrm{TeV}$ using the ATLAS detector, JHEP 09 (2013) 076 [arXiv: 1306.4945] [INSPIRE].

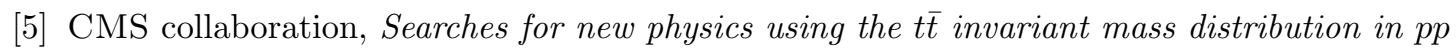
collisions at $\sqrt{s}=8 \mathrm{TeV}$, Phys. Rev. Lett. 111 (2013) 211804 [arXiv:1309.2030] [INSPIRE].

[6] CMS collaboration, Search for anomalous t $\bar{t}$ production in the highly-boosted all-hadronic final state, JHEP 09 (2012) 029 [Erratum ibid. 1403 (2014) 132] [arXiv:1204.2488] [INSPIRE]. 
[7] CMS collaboration, Search for massive resonances in dijet systems containing jets tagged as $W$ or $Z$ boson decays in pp collisions at $\sqrt{s}=8$ TeV, JHEP 08 (2014) 173 [arXiv: 1405.1994] [INSPIRE].

[8] CMS collaboration, Search for heavy resonances in the W/Z-tagged dijet mass spectrum in pp collisions at 7 TeV, Phys. Lett. B 723 (2013) 280 [arXiv:1212.1910] [InSPIRE].

[9] CMS collaboration, Search for massive resonances decaying into pairs of boosted bosons in semi-leptonic final states at $\sqrt{s}=8 \mathrm{TeV}$, JHEP 08 (2014) 174 [arXiv:1405.3447] [INSPIRE].

[10] CMS collaboration, Search for exotic resonances decaying into WZ/ZZ in pp collisions at $\sqrt{s}=7 \mathrm{TeV}$, JHEP 02 (2012) 036 [arXiv: 1211.5779] [INSPIRE].

[11] CMS collaboration, The CMS experiment at the CERN LHC, 2008 JINST 3 S08004 [INSPIRE].

[12] CMS collaboration, Jet performance in pp collisions at 7 TeV, CMS-PAS-JME-10-003 (2010).

[13] CMS collaboration, Particle-flow event reconstruction in CMS and performance for jets, taus and MET, CMS-PAS-PFT-09-001 (2009).

[14] CMS collaboration, Commissioning of the Particle-flow Event Reconstruction with the first LHC collisions recorded in the CMS detector, CMS-PAS-PFT-10-001 (2010).

[15] CMS collaboration, Energy calibration and resolution of the CMS electromagnetic calorimeter in pp collisions at $\sqrt{s}=7$ TeV, 2013 JINST 8 P09009 [arXiv:1306.2016] [INSPIRE].

[16] M. Cacciari, G.P. Salam and G. Soyez, The anti- $k_{t}$ jet clustering algorithm, JHEP 04 (2008) 063 [arXiv:0802.1189] [INSPIRE].

[17] M. Wobisch and T. Wengler, Hadronization corrections to jet cross-sections in deep inelastic scattering, hep-ph/9907280 [INSPIRE].

[18] Y.L. Dokshitzer, G.D. Leder, S. Moretti and B.R. Webber, Better jet clustering algorithms, JHEP 08 (1997) 001 [hep-ph/9707323] [INSPIRE].

[19] M. Cacciari, G.P. Salam and G. Soyez, FastJet user manual, Eur. Phys. J. C 72 (2012) 1896 [arXiv:1111.6097] [INSPIRE].

[20] CMS collaboration, Pileup removal algorithms, CMS-PAS-JME-14-001 (2014).

[21] M. Cacciari, G.P. Salam and G. Soyez, The catchment area of jets, JHEP 04 (2008) 005 [arXiv:0802.1188] [INSPIRE].

[22] M. Cacciari and G.P. Salam, Pileup subtraction using jet areas, Phys. Lett. B 659 (2008) 119 [arXiv:0707.1378] [INSPIRE].

[23] CMS collaboration, Determination of jet energy calibration and transverse momentum resolution in CMS, 2011 JINST $6 \mathrm{P} 11002$.

[24] CMS collaboration, Performance of CMS muon reconstruction in pp collision events at $\sqrt{s}=7$ TeV, $2012 \operatorname{JINST} 7 \mathrm{P} 10002$.

[25] CMS collaboration, Electron reconstruction and identification at $\sqrt{s}=7 \mathrm{Te} V$, CMS-PAS-EGM-10-004 (2010).

[26] CMS collaboration, CMS luminosity based on pixel cluster counting — Summer 2013 update, CMS-PAS-LUM-13-001 (2013). 
[27] K. Agashe, H. Davoudiasl, G. Perez and A. Soni, Warped gravitons at the LHC and beyond, Phys. Rev. D 76 (2007) 036006 [hep-ph/0701186] [InSPIRE].

[28] O. Antipin, D. Atwood and A. Soni, Search for RS gravitons via $W_{L} W_{L}$ decays, Phys. Lett. B 666 (2008) 155 [arXiv:0711.3175] [INSPIRE].

[29] O. Antipin and A. Soni, Towards establishing the spin of warped gravitons, JHEP 10 (2008) 018 [arXiv: 0806.3427] [INSPIRE].

[30] Y. Gao et al., Spin determination of single-produced resonances at hadron colliders, Phys. Rev. D 81 (2010) 075022 [arXiv: 1001.3396] [INSPIRE].

[31] S. Bolognesi et al., On the spin and parity of a single-produced resonance at the LHC, Phys. Rev. D 86 (2012) 095031 [arXiv:1208.4018] [INSPIRE].

[32] T. Sjöstrand, S. Mrenna and P.Z. Skands, PYTHIA 6.4 physics and manual, JHEP 05 (2006) 026 [hep-ph/0603175] [INSPIRE].

[33] CMS collaboration, Study of the underlying event at forward rapidity in pp collisions at $\sqrt{s}=0.9,2.76$ and $7 \mathrm{TeV}$, JHEP 04 (2013) 072 [arXiv: 1302.2394] [INSPIRE].

[34] P. Nason, A new method for combining NLO QCD with shower Monte Carlo algorithms, JHEP 11 (2004) 040 [hep-ph/0409146] [INSPIRE].

[35] S. Frixione, P. Nason and C. Oleari, Matching NLO QCD computations with parton shower simulations: the POWHEG method, JHEP 11 (2007) 070 [arXiv: 0709.2092] [INSPIRE].

[36] S. Alioli, P. Nason, C. Oleari and E. Re, A general framework for implementing NLO calculations in shower Monte Carlo programs: the POWHEG BOX, JHEP 06 (2010) 043 [arXiv: 1002.2581] [INSPIRE].

[37] J. Alwall et al., The automated computation of tree-level and next-to-leading order differential cross sections and their matching to parton shower simulations, JHEP 07 (2014) 079 [arXiv: 1405.0301] [INSPIRE].

[38] S. Gieseke et al., HERWIG++ 2.5 release note, arXiv:1102.1672 [INSPIRE].

[39] T. Sjöstrand, S. Mrenna and P.Z. Skands, A brief introduction to PYTHIA 8.1, Comput. Phys. Commun. 178 (2008) 852 [arXiv:0710.3820] [INSPIRE].

[40] J. Pumplin et al., New generation of parton distributions with uncertainties from global QCD analysis, JHEP 07 (2002) 012 [hep-ph/0201195] [INSPIRE].

[41] A.D. Martin, R.G. Roberts, W.J. Stirling and R.S. Thorne, MRST2001: partons and $\alpha_{s}$ from precise deep inelastic scattering and Tevatron jet data, Eur. Phys. J. C 23 (2002) 73 [hep-ph/0110215] [INSPIRE].

[42] H.-L. Lai et al., New parton distributions for collider physics, Phys. Rev. D 82 (2010) 074024 [arXiv: 1007.2241] [INSPIRE].

[43] S. Frixione and B.R. Webber, Matching NLO QCD computations and parton shower simulations, JHEP 06 (2002) 029 [hep-ph/0204244] [INSPIRE].

[44] GEANT4 collaboration, S. Agostinelli et al., GEANT4 - A simulation toolkit, Nucl. Instrum. Meth. A 506 (2003) 250 [INSPIRE].

[45] CMS collaboration, Identification of b-quark jets with the CMS experiment, 2013 JINST 8 P04013 [arXiv: 1211.4462] [INSPIRE]. 
[46] M. Gouzevitch et al., Scale-invariant resonance tagging in multijet events and new physics in Higgs pair production, JHEP 07 (2013) 148 [arXiv: 1303.6636] [INSPIRE].

[47] CMS collaboration, A Cambridge-Aachen (C-A) based Jet Algorithm for boosted top-jet tagging, CMS-PAS-JME-09-001 (2009).

[48] J.M. Butterworth, A.R. Davison, M. Rubin and G.P. Salam, Jet substructure as a new Higgs search channel at the LHC, Phys. Rev. Lett. 100 (2008) 242001 [arXiv:0802.2470] [INSPIRE].

[49] D. Krohn, J. Thaler and L.-T. Wang, Jet trimming, JHEP 02 (2010) 084 [arXiv:0912.1342] [INSPIRE].

[50] S.D. Ellis, C.K. Vermilion and J.R. Walsh, Techniques for improved heavy particle searches with jet substructure, Phys. Rev. D 80 (2009) 051501 [arXiv:0903.5081] [INSPIRE].

[51] S.D. Ellis, C.K. Vermilion and J.R. Walsh, Recombination algorithms and jet substructure: pruning as a tool for heavy particle searches, Phys. Rev. D 81 (2010) 094023 [arXiv: 0912.0033] [INSPIRE].

[52] J. Thaler and K. Van Tilburg, Identifying boosted objects with $N$-subjettiness, JHEP 03 (2011) 015 [arXiv: 1011.2268] [INSPIRE].

[53] S.D. Ellis and D.E. Soper, Successive combination jet algorithm for hadron collisions, Phys. Rev. D 48 (1993) 3160 [hep-ph/9305266] [INSPIRE].

[54] S.D. Ellis, A. Hornig, T.S. Roy, D. Krohn and M.D. Schwartz, Qjets: a non-deterministic approach to tree-based jet substructure, Phys. Rev. Lett. 108 (2012) 182003 [arXiv:1201.1914] [INSPIRE].

[55] A.J. Larkoski, G.P. Salam and J. Thaler, Energy correlation functions for jet substructure, JHEP 06 (2013) 108 [arXiv: 1305.0007] [INSPIRE].

[56] Y. Cui, Z. Han and M.D. Schwartz, W-jet tagging: optimizing the identification of boosted hadronically-decaying W bosons, Phys. Rev. D 83 (2011) 074023 [arXiv:1012.2077] [INSPIRE].

[57] D. Krohn, M.D. Schwartz, T. Lin and W.J. Waalewijn, Jet charge at the LHC, Phys. Rev. Lett. 110 (2013) 212001 [arXiv: 1209.2421] [INSPIRE].

[58] A. Hocker et al., TMVA - Toolkit for multivariate data analysis, PoS (ACAT) 040 [physics/0703039] [INSPIRE].

[59] CMS collaboration, V tagging observables and correlations, CMS-PAS-JME-14-002 (2014).

[60] CMS collaboration, Measurement of $W^{+} W^{-}$and $Z Z$ production cross sections in $p p$ collisions at $\sqrt{s}=8 \mathrm{TeV}$, Phys. Lett. B 721 (2013) 190 [arXiv:1301.4698] [InSPIRE].

[61] ATLAS collaboration, Charged particle multiplicities in $p p$ interactions at $\sqrt{s}=0.9$ and 7 $T e V$ in a diffractive limited phase-space measured with the ATLAS detector at the LHC and new PYTHIA6 tune, ATLAS-CONF-2010-031 (2010).

[62] ATLAS collaboration, ATLAS tunes of PYTHIA6 and Pythia 8 for MC11, ATLAS-PHYS-PUB-2011-009 (2011).

[63] CMS collaboration, Measurement of the W-boson helicity in top-quark decays from $t \bar{t}$ production in lepton + jets events in pp collisions at $\sqrt{s}=7 \mathrm{TeV}$, JHEP 10 (2013) 167 [arXiv:1308.3879] [INSPIRE]. 
[64] CMS collaboration, Jet energy corrections and uncertainties. Detector performance plots for 2012, CERN-CMS-DP-2012/012 (2012).

[65] CMS collaboration, Measurement of the inelastic proton-proton cross section at $\sqrt{s}=7 \mathrm{TeV}$, Phys. Lett. B 722 (2013) 5 [arXiv: 1210.6718] [INSPIRE]. 


\section{The CMS collaboration}

Yerevan Physics Institute, Yerevan, Armenia

V. Khachatryan, A.M. Sirunyan, A. Tumasyan

Institut für Hochenergiephysik der OeAW, Wien, Austria

W. Adam, T. Bergauer, M. Dragicevic, J. Erö, C. Fabjan ${ }^{1}$, M. Friedl, R. Frühwirth ${ }^{1}$, V.M. Ghete, C. Hartl, N. Hörmann, J. Hrubec, M. Jeitler ${ }^{1}$, W. Kiesenhofer, V. Knünz, M. Krammer ${ }^{1}$, I. Krätschmer, D. Liko, I. Mikulec, D. Rabady² , B. Rahbaran, H. Rohringer, R. Schöfbeck, J. Strauss, A. Taurok, W. Treberer-Treberspurg, W. Waltenberger, C.E. Wulz ${ }^{1}$

National Centre for Particle and High Energy Physics, Minsk, Belarus

V. Mossolov, N. Shumeiko, J. Suarez Gonzalez

\section{Universiteit Antwerpen, Antwerpen, Belgium}

S. Alderweireldt, M. Bansal, S. Bansal, T. Cornelis, E.A. De Wolf, X. Janssen, A. Knutsson, S. Luyckx, S. Ochesanu, B. Roland, R. Rougny, M. Van De Klundert, H. Van Haevermaet, P. Van Mechelen, N. Van Remortel, A. Van Spilbeeck

\section{Vrije Universiteit Brussel, Brussel, Belgium}

F. Blekman, S. Blyweert, J. D'Hondt, N. Daci, N. Heracleous, J. Keaveney, S. Lowette, M. Maes, A. Olbrechts, Q. Python, D. Strom, S. Tavernier, W. Van Doninck, P. Van Mulders, G.P. Van Onsem, I. Villella

Université Libre de Bruxelles, Bruxelles, Belgium

C. Caillol, B. Clerbaux, G. De Lentdecker, D. Dobur, L. Favart, A.P.R. Gay, A. Grebenyuk, A. Léonard, A. Mohammadi, L. Perniè ${ }^{2}$, T. Reis, T. Seva, L. Thomas, C. Vander Velde, P. Vanlaer, J. Wang

\section{Ghent University, Ghent, Belgium}

V. Adler, K. Beernaert, L. Benucci, A. Cimmino, S. Costantini, S. Crucy, S. Dildick, A. Fagot, G. Garcia, J. Mccartin, A.A. Ocampo Rios, D. Ryckbosch, S. Salva Diblen, M. Sigamani, N. Strobbe, F. Thyssen, M. Tytgat, E. Yazgan, N. Zaganidis

\section{Université Catholique de Louvain, Louvain-la-Neuve, Belgium}

S. Basegmez, C. Beluffi ${ }^{3}$, G. Bruno, R. Castello, A. Caudron, L. Ceard, G.G. Da Silveira, C. Delaere, T. du Pree, D. Favart, L. Forthomme, A. Giammanco ${ }^{4}$, J. Hollar, A. Jafari, P. Jez, M. Komm, V. Lemaitre, C. Nuttens, D. Pagano, L. Perrini, A. Pin, K. Piotrzkowski, A. Popov ${ }^{5}$, L. Quertenmont, M. Selvaggi, M. Vidal Marono, J.M. Vizan Garcia

\section{Université de Mons, Mons, Belgium}

N. Beliy, T. Caebergs, E. Daubie, G.H. Hammad

Centro Brasileiro de Pesquisas Fisicas, Rio de Janeiro, Brazil

W.L. Aldá Júnior, G.A. Alves, L. Brito, M. Correa Martins Junior, T. Dos Reis Martins, C. Mora Herrera, M.E. Pol 
Universidade do Estado do Rio de Janeiro, Rio de Janeiro, Brazil

W. Carvalho, J. Chinellato ${ }^{6}$, A. Custódio, E.M. Da Costa, D. De Jesus Damiao, C. De Oliveira Martins, S. Fonseca De Souza, H. Malbouisson, D. Matos Figueiredo, L. Mundim, H. Nogima, W.L. Prado Da Silva, J. Santaolalla, A. Santoro, A. Sznajder, E.J. Tonelli Manganote $^{6}$, A. Vilela Pereira

Universidade Estadual Paulista $^{a}$, Universidade Federal do ABC ${ }^{b}$, São Paulo, Brazil

C.A. Bernardes ${ }^{b}$, S. Dogra ${ }^{a}$, T.R. Fernandez Perez Tomei ${ }^{a}$, E.M. Gregores ${ }^{b}$, P.G. Mercadante ${ }^{b}$, S.F. Novaes ${ }^{a}$, Sandra S. Padula ${ }^{a}$

Institute for Nuclear Research and Nuclear Energy, Sofia, Bulgaria

A. Aleksandrov, V. Genchev², P. Iaydjiev, A. Marinov, S. Piperov, M. Rodozov, S. Stoykova, G. Sultanov, V. Tcholakov, M. Vutova

University of Sofia, Sofia, Bulgaria

A. Dimitrov, I. Glushkov, R. Hadjiiska, V. Kozhuharov, L. Litov, B. Pavlov, P. Petkov

Institute of High Energy Physics, Beijing, China

J.G. Bian, G.M. Chen, H.S. Chen, M. Chen, R. Du, C.H. Jiang, S. Liang, R. Plestina ${ }^{7}$, J. Tao, X. Wang, Z. Wang

State Key Laboratory of Nuclear Physics and Technology, Peking University, Beijing, China

C. Asawatangtrakuldee, Y. Ban, Y. Guo, Q. Li, S. Liu, Y. Mao, S.J. Qian, H. Teng,

D. Wang, W. Zou

Universidad de Los Andes, Bogota, Colombia

C. Avila, L.F. Chaparro Sierra, C. Florez, J.P. Gomez, B. Gomez Moreno, J.C. Sanabria

University of Split, Faculty of Electrical Engineering, Mechanical Engineering and Naval Architecture, Split, Croatia

N. Godinovic, D. Lelas, D. Polic, I. Puljak

University of Split, Faculty of Science, Split, Croatia

Z. Antunovic, M. Kovac

Institute Rudjer Boskovic, Zagreb, Croatia

V. Brigljevic, K. Kadija, J. Luetic, D. Mekterovic, L. Sudic

University of Cyprus, Nicosia, Cyprus

A. Attikis, G. Mavromanolakis, J. Mousa, C. Nicolaou, F. Ptochos, P.A. Razis

Charles University, Prague, Czech Republic

M. Bodlak, M. Finger, M. Finger Jr. ${ }^{8}$

Academy of Scientific Research and Technology of the Arab Republic of Egypt, Egyptian Network of High Energy Physics, Cairo, Egypt

Y. Assran ${ }^{9}$, A. Ellithi Kamel ${ }^{10}$, M.A. Mahmoud ${ }^{11}$, A. Radi ${ }^{12,13}$ 
National Institute of Chemical Physics and Biophysics, Tallinn, Estonia

M. Kadastik, M. Murumaa, M. Raidal, A. Tiko

Department of Physics, University of Helsinki, Helsinki, Finland

P. Eerola, G. Fedi, M. Voutilainen

Helsinki Institute of Physics, Helsinki, Finland

J. Härkönen, V. Karimäki, R. Kinnunen, M.J. Kortelainen, T. Lampén, K. Lassila-Perini,

S. Lehti, T. Lindén, P. Luukka, T. Mäenpää, T. Peltola, E. Tuominen, J. Tuominiemi,

E. Tuovinen, L. Wendland

Lappeenranta University of Technology, Lappeenranta, Finland

J. Talvitie, T. Tuuva

DSM/IRFU, CEA/Saclay, Gif-sur-Yvette, France

M. Besancon, F. Couderc, M. Dejardin, D. Denegri, B. Fabbro, J.L. Faure, C. Favaro,

F. Ferri, S. Ganjour, A. Givernaud, P. Gras, G. Hamel de Monchenault, P. Jarry, E. Locci,

J. Malcles, J. Rander, A. Rosowsky, M. Titov

Laboratoire Leprince-Ringuet, Ecole Polytechnique, IN2P3-CNRS, Palaiseau, France

S. Baffioni, F. Beaudette, P. Busson, C. Charlot, T. Dahms, M. Dalchenko, L. Dobrzynski, N. Filipovic, A. Florent, R. Granier de Cassagnac, L. Mastrolorenzo, P. Miné, C. Mironov, I.N. Naranjo, M. Nguyen, C. Ochando, P. Paganini, S. Regnard, R. Salerno, J.B. Sauvan, Y. Sirois, C. Veelken, Y. Yilmaz, A. Zabi

Institut Pluridisciplinaire Hubert Curien, Université de Strasbourg, Université de Haute Alsace Mulhouse, CNRS/IN2P3, Strasbourg, France

J.-L. Agram ${ }^{14}$, J. Andrea, A. Aubin, D. Bloch, J.-M. Brom, E.C. Chabert, C. Collard, E. Conte ${ }^{14}$, J.-C. Fontaine ${ }^{14}$, D. Gelé, U. Goerlach, C. Goetzmann, A.-C. Le Bihan, P. Van Hove

Centre de Calcul de l'Institut National de Physique Nucleaire et de Physique des Particules, CNRS/IN2P3, Villeurbanne, France

S. Gadrat

Université de Lyon, Université Claude Bernard Lyon 1, CNRS-IN2P3, Institut de Physique Nucléaire de Lyon, Villeurbanne, France

S. Beauceron, N. Beaupere, G. Boudoul'2, E. Bouvier, S. Brochet, C.A. Carrillo Montoya,

J. Chasserat, R. Chierici, D. Contardo², P. Depasse, H. El Mamouni, J. Fan, J. Fay,

S. Gascon, M. Gouzevitch, C. Guichardant, B. Ille, T. Kurca, M. Lethuillier, L. Mirabito,

S. Perries, J.D. Ruiz Alvarez, D. Sabes, L. Sgandurra, V. Sordini, M. Vander Donckt,

P. Verdier, S. Viret, H. Xiao

Institute of High Energy Physics and Informatization, Tbilisi State University, Tbilisi, Georgia

Z. Tsamalaidze ${ }^{8}$ 


\section{RWTH Aachen University, I. Physikalisches Institut, Aachen, Germany}

C. Autermann, S. Beranek, M. Bontenackels, M. Edelhoff, L. Feld, O. Hindrichs, K. Klein, A. Ostapchuk, A. Perieanu, F. Raupach, J. Sammet, S. Schael, H. Weber, B. Wittmer, V. Zhukov ${ }^{5}$

\section{RWTH Aachen University, III. Physikalisches Institut A, Aachen, Germany}

M. Ata, M. Brodski, E. Dietz-Laursonn, D. Duchardt, M. Erdmann, R. Fischer, A. Güth, T. Hebbeker, C. Heidemann, K. Hoepfner, D. Klingebiel, S. Knutzen, P. Kreuzer, M. Merschmeyer, A. Meyer, P. Millet, M. Olschewski, K. Padeken, P. Papacz, H. Reithler, S.A. Schmitz, L. Sonnenschein, D. Teyssier, S. Thüer, M. Weber

\section{RWTH Aachen University, III. Physikalisches Institut B, Aachen, Germany}

V. Cherepanov, Y. Erdogan, G. Flügge, H. Geenen, M. Geisler, W. Haj Ahmad, A. Heister,

F. Hoehle, B. Kargoll, T. Kress, Y. Kuessel, J. Lingemann², A. Nowack, I.M. Nugent, L. Perchalla, O. Pooth, A. Stahl

\section{Deutsches Elektronen-Synchrotron, Hamburg, Germany}

I. Asin, N. Bartosik, J. Behr, W. Behrenhoff, U. Behrens, A.J. Bell, M. Bergholz ${ }^{15}$, A. Bethani, K. Borras, A. Burgmeier, A. Cakir, L. Calligaris, A. Campbell, S. Choudhury, F. Costanza, C. Diez Pardos, S. Dooling, T. Dorland, G. Eckerlin, D. Eckstein, T. Eichhorn, G. Flucke, J. Garay Garcia, A. Geiser, P. Gunnellini, J. Hauk, M. Hempel, D. Horton, H. Jung, A. Kalogeropoulos, M. Kasemann, P. Katsas, J. Kieseler, C. Kleinwort, D. Krücker, W. Lange, J. Leonard, K. Lipka, A. Lobanov, W. Lohmann ${ }^{15}$, B. Lutz, R. Mankel, I. Marfin, I.-A. Melzer-Pellmann, A.B. Meyer, G. Mittag, J. Mnich, A. Mussgiller, S. Naumann-Emme, A. Nayak, O. Novgorodova, E. Ntomari, H. Perrey, D. Pitzl, R. Placakyte, A. Raspereza, P.M. Ribeiro Cipriano, E. Ron, M.Ö. Sahin, J. Salfeld-Nebgen, P. Saxena, R. Schmidt ${ }^{15}$, T. Schoerner-Sadenius, M. Schröder, C. Seitz, S. Spannagel, A.D.R. Vargas Trevino, R. Walsh, C. Wissing

\section{University of Hamburg, Hamburg, Germany}

M. Aldaya Martin, V. Blobel, M. Centis Vignali, A.R. Draeger, J. Erfle, E. Garutti, K. Goebel, M. Görner, J. Haller, M. Hoffmann, R.S. Höing, H. Kirschenmann, R. Klanner, R. Kogler, J. Lange, T. Lapsien, T. Lenz, I. Marchesini, J. Ott, T. Peiffer, N. Pietsch, J. Poehlsen, T. Poehlsen, D. Rathjens, C. Sander, H. Schettler, P. Schleper, E. Schlieckau, A. Schmidt, M. Seidel, V. Sola, H. Stadie, G. Steinbrück, D. Troendle, E. Usai, L. Vanelderen, A. Vanhoefer

\section{Institut für Experimentelle Kernphysik, Karlsruhe, Germany}

C. Barth, C. Baus, J. Berger, C. Böser, E. Butz, T. Chwalek, W. De Boer, A. Descroix, A. Dierlamm, M. Feindt, F. Frensch, M. Giffels, F. Hartmann², T. Hauth ${ }^{2}$, U. Husemann, I. Katkov ${ }^{5}$, A. Kornmayer ${ }^{2}$, E. Kuznetsova, P. Lobelle Pardo, M.U. Mozer, Th. Müller, A. Nürnberg, G. Quast, K. Rabbertz, F. Ratnikov, S. Röcker, H.J. Simonis, F.M. Stober, R. Ulrich, J. Wagner-Kuhr, S. Wayand, T. Weiler, R. Wolf 
Institute of Nuclear and Particle Physics (INPP), NCSR Demokritos, Aghia Paraskevi, Greece

G. Anagnostou, G. Daskalakis, T. Geralis, V.A. Giakoumopoulou, A. Kyriakis, D. Loukas, A. Markou, C. Markou, A. Psallidas, I. Topsis-Giotis

University of Athens, Athens, Greece

A. Agapitos, S. Kesisoglou, A. Panagiotou, N. Saoulidou, E. Stiliaris

University of Ioánnina, Ioánnina, Greece

X. Aslanoglou, I. Evangelou, G. Flouris, C. Foudas, P. Kokkas, N. Manthos, I. Papadopoulos, E. Paradas

Wigner Research Centre for Physics, Budapest, Hungary

G. Bencze, C. Hajdu, P. Hidas, D. Horvath ${ }^{16}$, F. Sikler, V. Veszpremi, G. Vesztergombi ${ }^{17}$, A.J. Zsigmond

Institute of Nuclear Research ATOMKI, Debrecen, Hungary

N. Beni, S. Czellar, J. Karancsi ${ }^{18}$, J. Molnar, J. Palinkas, Z. Szillasi

University of Debrecen, Debrecen, Hungary

P. Raics, Z.L. Trocsanyi, B. Ujvari

National Institute of Science Education and Research, Bhubaneswar, India

S.K. Swain

Panjab University, Chandigarh, India

S.B. Beri, V. Bhatnagar, R. Gupta, U.Bhawandeep, A.K. Kalsi, M. Kaur, M. Mittal, N. Nishu, J.B. Singh

University of Delhi, Delhi, India

Ashok Kumar, Arun Kumar, S. Ahuja, A. Bhardwaj, B.C. Choudhary, A. Kumar, S. Malhotra, M. Naimuddin, K. Ranjan, V. Sharma

Saha Institute of Nuclear Physics, Kolkata, India

S. Banerjee, S. Bhattacharya, K. Chatterjee, S. Dutta, B. Gomber, Sa. Jain, Sh. Jain, R. Khurana, A. Modak, S. Mukherjee, D. Roy, S. Sarkar, M. Sharan

Bhabha Atomic Research Centre, Mumbai, India

A. Abdulsalam, D. Dutta, S. Kailas, V. Kumar, A.K. Mohanty ${ }^{2}$, L.M. Pant, P. Shukla, A. Topkar

Tata Institute of Fundamental Research, Mumbai, India

T. Aziz, S. Banerjee, S. Bhowmik ${ }^{19}$, R.M. Chatterjee, R.K. Dewanjee, S. Dugad, S. Ganguly, S. Ghosh, M. Guchait, A. Gurtu ${ }^{20}$, G. Kole, S. Kumar, M. Maity ${ }^{19}$, G. Majumder, K. Mazumdar, G.B. Mohanty, B. Parida, K. Sudhakar, N. Wickramage ${ }^{21}$

Institute for Research in Fundamental Sciences (IPM), Tehran, Iran

H. Bakhshiansohi, H. Behnamian, S.M. Etesami ${ }^{22}$, A. Fahim ${ }^{23}$, R. Goldouzian, M. Khakzad, M. Mohammadi Najafabadi, M. Naseri, S. Paktinat Mehdiabadi, F. Rezaei Hosseinabadi, B. Safarzadeh ${ }^{24}$, M. Zeinali 
University College Dublin, Dublin, Ireland

M. Felcini, M. Grunewald

INFN Sezione di Bari ${ }^{a}$, Università di Bari ${ }^{b}$, Politecnico di Bari ${ }^{c}$, Bari, Italy

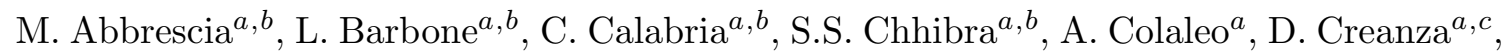
N. De Filippis ${ }^{a, c}$, M. De Palma ${ }^{a, b}$, L. Fiore ${ }^{a}$, G. Iaselli ${ }^{a, c}$, G. Maggi ${ }^{a, c}$, M. Maggi $^{a}, \mathrm{~S} \mathrm{My}^{a, c}$, S. Nuzzo ${ }^{a, b}$, A. Pompili ${ }^{a, b}$, G. Pugliese ${ }^{a, c}$, R. Radogna ${ }^{a, b, 2}$, G. Selvaggi ${ }^{a, b}$, L. Silvestris ${ }^{a, 2}$, G. Singh ${ }^{a, b}$, R. Venditti ${ }^{a}, b$, P. Verwilligen ${ }^{a}$, G. Zito ${ }^{a}$

\section{INFN Sezione di Bologna ${ }^{a}$, Università di Bologna ${ }^{b}$, Bologna, Italy}

G. Abbiendi $^{a}$, A.C. Benvenuti ${ }^{a}$, D. Bonacorsi ${ }^{a}, b$, S. Braibant-Giacomelli ${ }^{a}, b$, L. Brigliadori ${ }^{a, b}$, R. Campanini ${ }^{a, b}$, P. Capiluppi ${ }^{a, b}$, A. Castro ${ }^{a, b}$, F.R. Cavallo ${ }^{a}$,

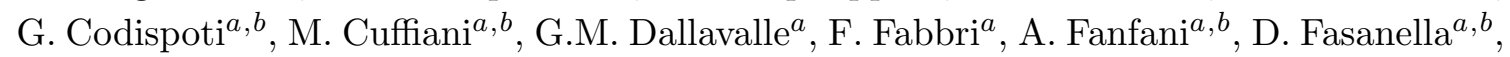
P. Giacomelli ${ }^{a}$, C. Grandi ${ }^{a}$, L. Guiducci ${ }^{a}, b$, S. Marcellini ${ }^{a}$, G. Masetti ${ }^{a, 2}$, A. Montanari ${ }^{a}$,

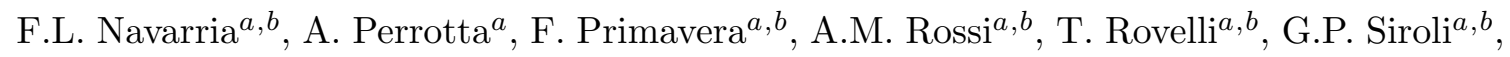
N. Tosi ${ }^{a, b}$, R. Travaglini ${ }^{a, b}$

INFN Sezione di Catania ${ }^{a}$, Università di Catania ${ }^{b}, \operatorname{CSFNSM}^{c}$, Catania, Italy S. Albergo ${ }^{a, b}$, G. Cappello ${ }^{a}$, M. Chiorboli ${ }^{a}, b$, S. Costa ${ }^{a, b}$, F. Giordano ${ }^{a, 2}$, R. Potenza ${ }^{a, b}$, A. Tricomi ${ }^{a, b}$, C. Tuve ${ }^{a, b}$

INFN Sezione di Firenze ${ }^{a}$, Università di Firenze ${ }^{b}$, Firenze, Italy

G. Barbagli ${ }^{a}$, V. Ciulli ${ }^{a, b}$, C. Civinini ${ }^{a}$, R. D'Alessandro ${ }^{a, b}$, E. Focardi ${ }^{a}, b$, E. Gallo ${ }^{a}$, S. Gonzi ${ }^{a, b}$, V. Gori ${ }^{a, b, 2}$, P. Lenzi ${ }^{a, b}$, M. Meschini ${ }^{a}$, S. Paoletti ${ }^{a}$, G. Sguazzoni ${ }^{a}$, A. Tropiano ${ }^{a, b}$

INFN Laboratori Nazionali di Frascati, Frascati, Italy

L. Benussi, S. Bianco, F. Fabbri, D. Piccolo

INFN Sezione di Genova ${ }^{a}$, Università di Genova ${ }^{b}$, Genova, Italy R. Ferretti ${ }^{a, b}$, F. Ferro $^{a}$, M. Lo Vetere ${ }^{a, b}$, E. Robutti ${ }^{a}$, S. Tosi ${ }^{a, b}$

INFN Sezione di Milano-Bicocca ${ }^{a}$, Università di Milano-Bicocca ${ }^{b}$, Milano, Italy

M.E. Dinardo ${ }^{a, b}$, S. Fiorendi ${ }^{a, b, 2}$, S. Gennai ${ }^{a, 2}$, R. Gerosa ${ }^{a, b, 2}$, A. Ghezzi ${ }^{a, b}$, P. Govoni ${ }^{a, b}$, M.T. Lucchini ${ }^{a, b, 2}$, S. Malvezzi ${ }^{a}$, R.A. Manzoni ${ }^{a, b}$, A. Martelli ${ }^{a, b}$, B. Marzocchi ${ }^{a, b}$,

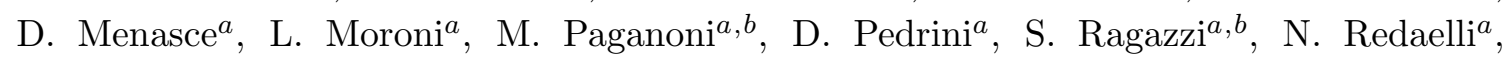
T. Tabarelli de Fatis ${ }^{a, b}$

INFN Sezione di Napoli ${ }^{a}$, Università di Napoli 'Federico II' ${ }^{b}$, Università della Basilicata (Potenza) ${ }^{c}$, Università G. Marconi (Roma) ${ }^{d}$, Napoli, Italy

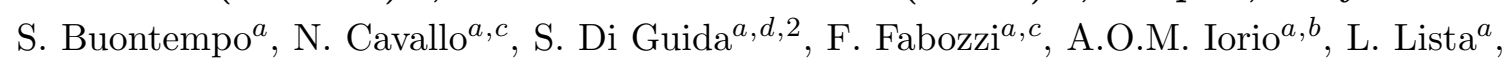
S. Meola ${ }^{a, d, 2}$, M. Merola ${ }^{a}$, P. Paolucci ${ }^{a, 2}$

INFN Sezione di Padova ${ }^{a}$, Università di Padova ${ }^{b}$, Università di Trento (Trento) ${ }^{c}$, Padova, Italy

P. Azzi ${ }^{a}$, N. Bacchetta ${ }^{a}$, D. Bisello ${ }^{a, b}$, A. Branca ${ }^{a, b}$, R. Carlin ${ }^{a, b}$, P. Checchia $^{a}$, M. Dall'Osso ${ }^{a, b}$, M. Galanti ${ }^{a, b}$, F. Gasparini ${ }^{a, b}$, U. Gasparini ${ }^{a, b}$, P. Giubilato ${ }^{a, b}$, 


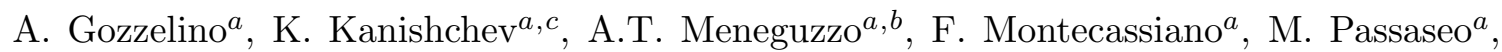

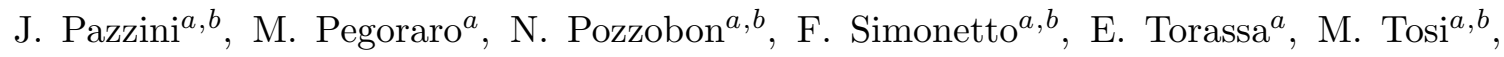

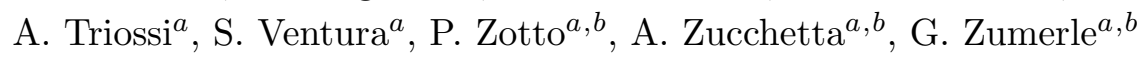

INFN Sezione di Pavia ${ }^{a}$, Università di Pavia ${ }^{b}$, Pavia, Italy

M. Gabusi ${ }^{a}, b$, S.P. Ratti ${ }^{a, b}$, C. Riccardi ${ }^{a, b}$, P. Salvini ${ }^{a}$, P. Vitulo ${ }^{a, b}$

INFN Sezione di Perugia ${ }^{a}$, Università di Perugia ${ }^{b}$, Perugia, Italy

M. Biasini ${ }^{a, b}$, G.M. Bilei ${ }^{a}$, D. Ciangottini ${ }^{a, b}$, L. Fanò ${ }^{a, b}$, P. Lariccia $^{a, b}$, G. Mantovani $^{a, b}$, M. Menichelli ${ }^{a}$, F. Romeo ${ }^{a, b}$, A. Saha ${ }^{a}$, A. Santocchia ${ }^{a, b}$, A. Spiezia ${ }^{a, b, 2}$

INFN Sezione di Pisa ${ }^{a}$, Università di Pisa ${ }^{b}$, Scuola Normale Superiore di Pisa ${ }^{c}$, Pisa, Italy

K. Androsov ${ }^{a}, 25$, P. Azzurri ${ }^{a}$, G. Bagliesi ${ }^{a}$, J. Bernardini ${ }^{a}$, T. Boccali ${ }^{a}$, G. Broccolo $^{a, c}$, R. Castaldi ${ }^{a}$, M.A. Ciocci ${ }^{a, 25}$, R. Dell'Orso ${ }^{a}$, S. Donato ${ }^{a, c}$, F. Fiori ${ }^{a, c}$, L. Foà $\grave{a}^{a, c}$,

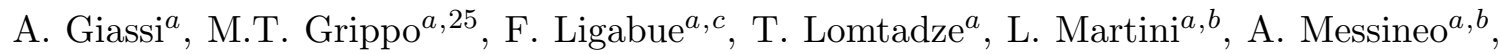
C.S. Moon ${ }^{a, 26}$, F. Palla ${ }^{a, 2}$, A. Rizzi ${ }^{a, b}$, A. Savoy-Navarro ${ }^{a, 27}$, A.T. $\operatorname{Serban}^{a}$, P. Spagnolo ${ }^{a}$,

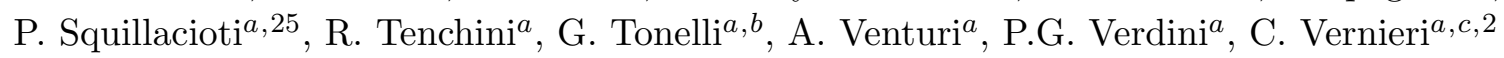

INFN Sezione di Roma ${ }^{a}$, Università di Roma ${ }^{b}$, Roma, Italy

L. Barone ${ }^{a, b}$, F. Cavallari ${ }^{a}$, G. D'imperio ${ }^{a, b}$, D. Del Re Re $^{a, b}$, M. Diemoz ${ }^{a}$, M. Grassi ${ }^{a, b}$, C. Jorda ${ }^{a}$, E. Longo ${ }^{a}, b$, F. Margaroli ${ }^{a, b}$, P. Meridiani ${ }^{a}$, F. Micheli ${ }^{a, b, 2}$, S. Nourbakhsh ${ }^{a, b}$, G. Organtini ${ }^{a, b}$, R. Paramatti ${ }^{a}$, S. Rahatlou ${ }^{a, b}$, C. Rovelli ${ }^{a}$, F. Santanastasio ${ }^{a, b}$, L. Soffi ${ }^{a, b, 2}$, P. Traczyk ${ }^{a, b}$

INFN Sezione di Torino ${ }^{a}$, Università di Torino ${ }^{b}$, Università del Piemonte Orientale (Novara) ${ }^{c}$, Torino, Italy

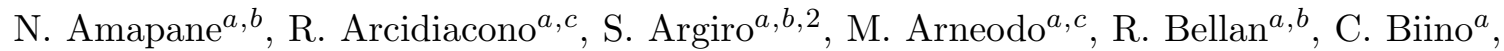

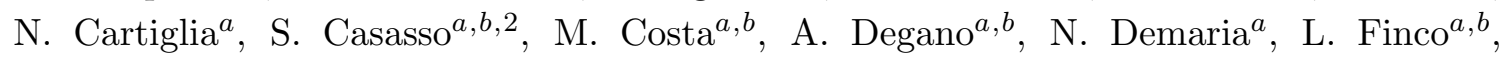
C. Mariotti ${ }^{a}$, S. Maselli ${ }^{a}$, E. Migliore ${ }^{a, b}$, V. Monaco ${ }^{a, b}$, M. Musich $^{a}$, M.M. Obertino ${ }^{a, c, 2}$, G. Ortona ${ }^{a, b}$, L. Pacher ${ }^{a, b}$, N. Pastrone ${ }^{a}$, M. Pelliccioni ${ }^{a}$, G.L. Pinna Angioni ${ }^{a, b}$, A. Potenza ${ }^{a, b}$, A. Romero ${ }^{a, b}$, M. Ruspa ${ }^{a, c}$, R. Sacchi ${ }^{a, b}$, A. Solano ${ }^{a, b}$, A. Staiano ${ }^{a}$, U. Tamponi ${ }^{a}$

INFN Sezione di Trieste ${ }^{a}$, Università di Trieste ${ }^{b}$, Trieste, Italy

S. Belforte ${ }^{a}$, V. Candelise ${ }^{a, b}$, M. Casarsa ${ }^{a}$, F. Cossutti ${ }^{a}$, G. Della Ricca ${ }^{a, b}$, B. Gobbo ${ }^{a}$,

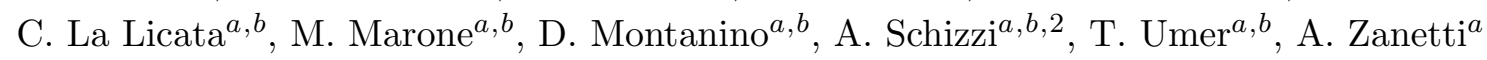

Kangwon National University, Chunchon, Korea

S. Chang, A. Kropivnitskaya, S.K. Nam

Kyungpook National University, Daegu, Korea

D.H. Kim, G.N. Kim, M.S. Kim, D.J. Kong, S. Lee, Y.D. Oh, H. Park, A. Sakharov, D.C. Son

Chonbuk National University, Jeonju, Korea

T.J. Kim 
Chonnam National University, Institute for Universe and Elementary Particles, Kwangju, Korea

J.Y. Kim, S. Song

Korea University, Seoul, Korea

S. Choi, D. Gyun, B. Hong, M. Jo, H. Kim, Y. Kim, B. Lee, K.S. Lee, S.K. Park, Y. Roh

University of Seoul, Seoul, Korea

M. Choi, J.H. Kim, I.C. Park, S. Park, G. Ryu, M.S. Ryu

Sungkyunkwan University, Suwon, Korea

Y. Choi, Y.K. Choi, J. Goh, D. Kim, E. Kwon, J. Lee, H. Seo, I. Yu

Vilnius University, Vilnius, Lithuania

A. Juodagalvis

National Centre for Particle Physics, Universiti Malaya, Kuala Lumpur, Malaysia

J.R. Komaragiri, M.A.B. Md Ali

Centro de Investigacion y de Estudios Avanzados del IPN, Mexico City, Mexico

H. Castilla-Valdez, E. De La Cruz-Burelo, I. Heredia-de La Cruz ${ }^{28}$, R. Lopez-Fernandez,

A. Sanchez-Hernandez

Universidad Iberoamericana, Mexico City, Mexico

S. Carrillo Moreno, F. Vazquez Valencia

Benemerita Universidad Autonoma de Puebla, Puebla, Mexico

I. Pedraza, H.A. Salazar Ibarguen

Universidad Autónoma de San Luis Potosí, San Luis Potosí, Mexico

E. Casimiro Linares, A. Morelos Pineda

University of Auckland, Auckland, New Zealand

D. Krofcheck

University of Canterbury, Christchurch, New Zealand

P.H. Butler, S. Reucroft

National Centre for Physics, Quaid-I-Azam University, Islamabad, Pakistan

A. Ahmad, M. Ahmad, Q. Hassan, H.R. Hoorani, S. Khalid, W.A. Khan, T. Khurshid, M.A. Shah, M. Shoaib

National Centre for Nuclear Research, Swierk, Poland

H. Bialkowska, M. Bluj, B. Boimska, T. Frueboes, M. Górski, M. Kazana, K. Nawrocki, K. Romanowska-Rybinska, M. Szleper, P. Zalewski

Institute of Experimental Physics, Faculty of Physics, University of Warsaw, Warsaw, Poland

G. Brona, K. Bunkowski, M. Cwiok, W. Dominik, K. Doroba, A. Kalinowski, M. Konecki, J. Krolikowski, M. Misiura, M. Olszewski, W. Wolszczak 
Laboratório de Instrumentação e Física Experimental de Partículas, Lisboa, Portugal

P. Bargassa, C. Beirão Da Cruz E Silva, P. Faccioli, P.G. Ferreira Parracho, M. Gallinaro, F. Nguyen, J. Rodrigues Antunes, J. Seixas, J. Varela, P. Vischia

Joint Institute for Nuclear Research, Dubna, Russia

S. Afanasiev, P. Bunin, I. Golutvin, I. Gorbunov, V. Karjavin, V. Konoplyanikov, G. Kozlov, A. Lanev, A. Malakhov, V. Matveev $^{29}$, P. Moisenz, V. Palichik, V. Perelygin, S. Shmatov, S. Shulha, N. Skatchkov, V. Smirnov, A. Zarubin

Petersburg Nuclear Physics Institute, Gatchina (St. Petersburg), Russia

V. Golovtsov, Y. Ivanov, V. Kim ${ }^{30}$, P. Levchenko, V. Murzin, V. Oreshkin, I. Smirnov, V. Sulimov, L. Uvarov, S. Vavilov, A. Vorobyev, An. Vorobyev

Institute for Nuclear Research, Moscow, Russia

Yu. Andreev, A. Dermenev, S. Gninenko, N. Golubev, M. Kirsanov, N. Krasnikov, A. Pashenkov, D. Tlisov, A. Toropin

Institute for Theoretical and Experimental Physics, Moscow, Russia

V. Epshteyn, V. Gavrilov, N. Lychkovskaya, V. Popov, G. Safronov, S. Semenov, A. Spiridonov, V. Stolin, E. Vlasov, A. Zhokin

P.N. Lebedev Physical Institute, Moscow, Russia

V. Andreev, M. Azarkin, I. Dremin, M. Kirakosyan, A. Leonidov, G. Mesyats, S.V. Rusakov, A. Vinogradov

Skobeltsyn Institute of Nuclear Physics, Lomonosov Moscow State University, Moscow, Russia

A. Belyaev, E. Boos, M. Dubinin ${ }^{31}$, L. Dudko, A. Ershov, A. Gribushin, A. Kaminskiy ${ }^{32}$, V. Klyukhin, O. Kodolova, I. Lokhtin, S. Obraztsov, S. Petrushanko, V. Savrin

State Research Center of Russian Federation, Institute for High Energy Physics, Protvino, Russia

I. Azhgirey, I. Bayshev, S. Bitioukov, V. Kachanov, A. Kalinin, D. Konstantinov, V. Krychkine, V. Petrov, R. Ryutin, A. Sobol, L. Tourtchanovitch, S. Troshin, N. Tyurin, A. Uzunian, A. Volkov

University of Belgrade, Faculty of Physics and Vinca Institute of Nuclear Sciences, Belgrade, Serbia

P. Adzic ${ }^{33}$, M. Ekmedzic, J. Milosevic, V. Rekovic

Centro de Investigaciones Energéticas Medioambientales y Tecnológicas (CIEMAT), Madrid, Spain

J. Alcaraz Maestre, C. Battilana, E. Calvo, M. Cerrada, M. Chamizo Llatas, N. Colino, B. De La Cruz, A. Delgado Peris, D. Domínguez Vázquez, A. Escalante Del Valle, C. Fernandez Bedoya, J.P. Fernández Ramos, J. Flix, M.C. Fouz, P. Garcia-Abia, O. Gonzalez Lopez, S. Goy Lopez, J.M. Hernandez, M.I. Josa, G. Merino, E. Navarro De Martino, 
A. Pérez-Calero Yzquierdo, J. Puerta Pelayo, A. Quintario Olmeda, I. Redondo, L. Romero, M.S. Soares

\section{Universidad Autónoma de Madrid, Madrid, Spain}

C. Albajar, J.F. de Trocóniz, M. Missiroli, D. Moran

\section{Universidad de Oviedo, Oviedo, Spain}

H. Brun, J. Cuevas, J. Fernandez Menendez, S. Folgueras, I. Gonzalez Caballero, L. Lloret Iglesias

\section{Instituto de Física de Cantabria (IFCA), CSIC-Universidad de Cantabria, Santander, Spain}

J.A. Brochero Cifuentes, I.J. Cabrillo, A. Calderon, J. Duarte Campderros, M. Fernandez, G. Gomez, A. Graziano, A. Lopez Virto, J. Marco, R. Marco, C. Martinez Rivero, F. Matorras, F.J. Munoz Sanchez, J. Piedra Gomez, T. Rodrigo, A.Y. Rodríguez-Marrero, A. Ruiz-Jimeno, L. Scodellaro, I. Vila, R. Vilar Cortabitarte

\section{CERN, European Organization for Nuclear Research, Geneva, Switzerland}

D. Abbaneo, E. Auffray, G. Auzinger, M. Bachtis, P. Baillon, A.H. Ball, D. Barney, A. Benaglia, J. Bendavid, L. Benhabib, J.F. Benitez, C. Bernet ${ }^{7}$, G. Bianchi, P. Bloch, A. Bocci, A. Bonato, O. Bondu, C. Botta, H. Breuker, T. Camporesi, G. Cerminara, S. Colafranceschi ${ }^{34}$, M. D'Alfonso, D. d'Enterria, A. Dabrowski, A. David, F. De Guio, A. De Roeck, S. De Visscher, M. Dobson, M. Dordevic, N. Dupont-Sagorin, A. ElliottPeisert, J. Eugster, G. Franzoni, W. Funk, D. Gigi, K. Gill, D. Giordano, M. Girone, F. Glege, R. Guida, S. Gundacker, M. Guthoff, J. Hammer, M. Hansen, P. Harris, J. Hegeman, V. Innocente, P. Janot, K. Kousouris, K. Krajczar, P. Lecoq, C. Lourenço, N. Magini, L. Malgeri, M. Mannelli, J. Marrouche, L. Masetti, F. Meijers, S. Mersi, E. Meschi, F. Moortgat, S. Morovic, M. Mulders, P. Musella, L. Orsini, L. Pape, E. Perez, L. Perrozzi, A. Petrilli, G. Petrucciani, A. Pfeiffer, M. Pierini, M. Pimiä, D. Piparo, M. Plagge, A. Racz, G. Rolandi ${ }^{35}$, M. Rovere, H. Sakulin, C. Schäfer, C. Schwick, A. Sharma, P. Siegrist, P. Silva, M. Simon, P. Sphicas ${ }^{36}$, D. Spiga, J. Steggemann, B. Stieger, M. Stoye, Y. Takahashi, D. Treille, A. Tsirou, G.I. Veres ${ }^{17}$, J.R. Vlimant, N. Wardle, H.K. Wöhri, H. Wollny, W.D. Zeuner

\section{Paul Scherrer Institut, Villigen, Switzerland}

W. Bertl, K. Deiters, W. Erdmann, R. Horisberger, Q. Ingram, H.C. Kaestli, D. Kotlinski, U. Langenegger, D. Renker, T. Rohe

\section{Institute for Particle Physics, ETH Zurich, Zurich, Switzerland}

F. Bachmair, L. Bäni, L. Bianchini, M.A. Buchmann, B. Casal, N. Chanon, A. Deisher, G. Dissertori, M. Dittmar, M. Donegà, M. Dünser, P. Eller, C. Grab, D. Hits, W. Lustermann, B. Mangano, A.C. Marini, P. Martinez Ruiz del Arbol, D. Meister, N. Mohr, C. Nägeli ${ }^{37}$, F. Nessi-Tedaldi, F. Pandolfi, F. Pauss, M. Peruzzi, M. Quittnat, L. Rebane, M. Rossini, A. Starodumov ${ }^{38}$, M. Takahashi, K. Theofilatos, R. Wallny, H.A. Weber 
Universität Zürich, Zurich, Switzerland

C. Amsler ${ }^{39}$, M.F. Canelli, V. Chiochia, A. De Cosa, A. Hinzmann, T. Hreus, B. Kilminster,

C. Lange, B. Millan Mejias, J. Ngadiuba, P. Robmann, F.J. Ronga, S. Taroni, M. Verzetti,

Y. Yang

National Central University, Chung-Li, Taiwan

M. Cardaci, K.H. Chen, C. Ferro, C.M. Kuo, W. Lin, Y.J. Lu, R. Volpe, S.S. Yu

National Taiwan University (NTU), Taipei, Taiwan

P. Chang, Y.H. Chang, Y.W. Chang, Y. Chao, K.F. Chen, P.H. Chen, C. Dietz, U. Grundler, W.-S. Hou, K.Y. Kao, Y.J. Lei, Y.F. Liu, R.-S. Lu, D. Majumder, E. Petrakou, Y.M. Tzeng, R. Wilken

Chulalongkorn University, Faculty of Science, Department of Physics, Bangkok, Thailand

B. Asavapibhop, N. Srimanobhas, N. Suwonjandee

Cukurova University, Adana, Turkey

A. Adiguzel, M.N. Bakirci ${ }^{40}$, S. Cerci ${ }^{41}$, C. Dozen, I. Dumanoglu, E. Eskut, S. Girgis, G. Gokbulut, E. Gurpinar, I. Hos, E.E. Kangal, A. Kayis Topaksu, G. Onengut ${ }^{42}$, K. Ozdemir, S. Ozturk ${ }^{40}$, A. Polatoz, D. Sunar Cerci ${ }^{41}$, B. Tali ${ }^{41}$, H. Topakli ${ }^{40}$, M. Vergili

Middle East Technical University, Physics Department, Ankara, Turkey

I.V. Akin, B. Bilin, S. Bilmis, H. Gamsizkan, G. Karapinar ${ }^{43}$, K. Ocalan, S. Sekmen, U.E. Surat, M. Yalvac, M. Zeyrek

Bogazici University, Istanbul, Turkey

E. Gülmez, B. Isildak ${ }^{44}$, M. Kaya ${ }^{45}$, O. Kaya ${ }^{46}$

Istanbul Technical University, Istanbul, Turkey

K. Cankocak, F.I. Vardarlı

National Scientific Center, Kharkov Institute of Physics and Technology, Kharkov, Ukraine

L. Levchuk, P. Sorokin

University of Bristol, Bristol, United Kingdom

J.J. Brooke, E. Clement, D. Cussans, H. Flacher, R. Frazier, J. Goldstein, M. Grimes, G.P. Heath, H.F. Heath, J. Jacob, L. Kreczko, C. Lucas, Z. Meng, D.M. Newbold ${ }^{47}$, S. Paramesvaran, A. Poll, S. Senkin, V.J. Smith, T. Williams

\section{Rutherford Appleton Laboratory, Didcot, United Kingdom}

K.W. Bell, A. Belyaev ${ }^{48}$, C. Brew, R.M. Brown, D.J.A. Cockerill, J.A. Coughlan, K. Harder, S. Harper, E. Olaiya, D. Petyt, C.H. Shepherd-Themistocleous, A. Thea, I.R. Tomalin, W.J. Womersley, S.D. Worm

\section{Imperial College, London, United Kingdom}

M. Baber, R. Bainbridge, O. Buchmuller, D. Burton, D. Colling, N. Cripps, M. Cutajar, P. Dauncey, G. Davies, M. Della Negra, P. Dunne, W. Ferguson, J. Fulcher, D. Futyan, 
A. Gilbert, G. Hall, G. Iles, M. Jarvis, G. Karapostoli, M. Kenzie, R. Lane, R. Lucas ${ }^{47}$, L. Lyons, A.-M. Magnan, S. Malik, B. Mathias, J. Nash, A. Nikitenko ${ }^{38}$, J. Pela, M. Pesaresi, K. Petridis, D.M. Raymond, S. Rogerson, A. Rose, C. Seez, P. Sharp ${ }^{\dagger}$, A. Tapper, M. Vazquez Acosta, T. Virdee, S.C. Zenz

\section{Brunel University, Uxbridge, United Kingdom}

J.E. Cole, P.R. Hobson, A. Khan, P. Kyberd, D. Leggat, D. Leslie, W. Martin, I.D. Reid, P. Symonds, L. Teodorescu, M. Turner

Baylor University, Waco, U.S.A.

J. Dittmann, K. Hatakeyama, A. Kasmi, H. Liu, T. Scarborough

The University of Alabama, Tuscaloosa, U.S.A.

O. Charaf, S.I. Cooper, C. Henderson, P. Rumerio

Boston University, Boston, U.S.A.

A. Avetisyan, T. Bose, C. Fantasia, P. Lawson, C. Richardson, J. Rohlf, J. St. John, L. Sulak

Brown University, Providence, U.S.A.

J. Alimena, E. Berry, S. Bhattacharya, G. Christopher, D. Cutts, Z. Demiragli, N. Dhingra, A. Ferapontov, A. Garabedian, U. Heintz, G. Kukartsev, E. Laird, G. Landsberg, M. Luk, M. Narain, M. Segala, T. Sinthuprasith, T. Speer, J. Swanson

University of California, Davis, Davis, U.S.A.

R. Breedon, G. Breto, M. Calderon De La Barca Sanchez, S. Chauhan, M. Chertok, J. Conway, R. Conway, P.T. Cox, R. Erbacher, M. Gardner, W. Ko, R. Lander, T. Miceli, M. Mulhearn, D. Pellett, J. Pilot, F. Ricci-Tam, M. Searle, S. Shalhout, J. Smith, M. Squires, D. Stolp, M. Tripathi, S. Wilbur, R. Yohay

University of California, Los Angeles, U.S.A.

R. Cousins, P. Everaerts, C. Farrell, J. Hauser, M. Ignatenko, G. Rakness, E. Takasugi, V. Valuev, M. Weber

University of California, Riverside, Riverside, U.S.A.

K. Burt, R. Clare, J. Ellison, J.W. Gary, G. Hanson, J. Heilman, M. Ivova Rikova, P. Jandir, E. Kennedy, F. Lacroix, O.R. Long, A. Luthra, M. Malberti, H. Nguyen, M. Olmedo Negrete, A. Shrinivas, S. Sumowidagdo, S. Wimpenny

University of California, San Diego, La Jolla, U.S.A.

W. Andrews, J.G. Branson, G.B. Cerati, S. Cittolin, R.T. D'Agnolo, D. Evans, A. Holzner, R. Kelley, D. Klein, M. Lebourgeois, J. Letts, I. Macneill, D. Olivito, S. Padhi, C. Palmer, M. Pieri, M. Sani, V. Sharma, S. Simon, E. Sudano, M. Tadel, Y. Tu, A. Vartak, C. Welke, F. Würthwein, A. Yagil, J. Yoo

University of California, Santa Barbara, Santa Barbara, U.S.A.

D. Barge, J. Bradmiller-Feld, C. Campagnari, T. Danielson, A. Dishaw, K. Flowers, M. Franco Sevilla, P. Geffert, C. George, F. Golf, L. Gouskos, J. Incandela, C. Justus, N. Mccoll, J. Richman, D. Stuart, W. To, C. West 
California Institute of Technology, Pasadena, U.S.A.

A. Apresyan, A. Bornheim, J. Bunn, Y. Chen, E. Di Marco, J. Duarte, A. Mott, H.B. Newman, C. Pena, C. Rogan, M. Spiropulu, V. Timciuc, R. Wilkinson, S. Xie, R.Y. Zhu

Carnegie Mellon University, Pittsburgh, U.S.A.

V. Azzolini, A. Calamba, B. Carlson, T. Ferguson, Y. Iiyama, M. Paulini, J. Russ, H. Vogel, I. Vorobiev

University of Colorado at Boulder, Boulder, U.S.A.

J.P. Cumalat, W.T. Ford, A. Gaz, E. Luiggi Lopez, U. Nauenberg, J.G. Smith, K. Stenson, K.A. Ulmer, S.R. Wagner

Cornell University, Ithaca, U.S.A.

J. Alexander, A. Chatterjee, J. Chu, S. Dittmer, N. Eggert, N. Mirman, G. Nicolas Kaufman, J.R. Patterson, A. Ryd, E. Salvati, L. Skinnari, W. Sun, W.D. Teo, J. Thom, J. Thompson, J. Tucker, Y. Weng, L. Winstrom, P. Wittich

Fairfield University, Fairfield, U.S.A.

D. Winn

Fermi National Accelerator Laboratory, Batavia, U.S.A.

S. Abdullin, M. Albrow, J. Anderson, G. Apollinari, L.A.T. Bauerdick, A. Beretvas, J. Berryhill, P.C. Bhat, K. Burkett, J.N. Butler, H.W.K. Cheung, F. Chlebana, S. Cihangir, V.D. Elvira, I. Fisk, J. Freeman, Y. Gao, E. Gottschalk, L. Gray, D. Green, S. Grünendahl, O. Gutsche, J. Hanlon, D. Hare, R.M. Harris, J. Hirschauer, B. Hooberman, S. Jindariani, M. Johnson, U. Joshi, K. Kaadze, B. Klima, B. Kreis, S. Kwan, J. Linacre, D. Lincoln, R. Lipton, T. Liu, J. Lykken, K. Maeshima, J.M. Marraffino, V.I. Martinez Outschoorn, S. Maruyama, D. Mason, P. McBride, K. Mishra, S. Mrenna, Y. Musienko ${ }^{29}$, S. Nahn, C. Newman-Holmes, V. O'Dell, O. Prokofyev, E. Sexton-Kennedy, S. Sharma, A. Soha, W.J. Spalding, L. Spiegel, L. Taylor, S. Tkaczyk, N.V. Tran, L. Uplegger, E.W. Vaandering, R. Vidal, A. Whitbeck, J. Whitmore, F. Yang

University of Florida, Gainesville, U.S.A.

D. Acosta, P. Avery, P. Bortignon, D. Bourilkov, M. Carver, T. Cheng, D. Curry, S. Das, M. De Gruttola, G.P. Di Giovanni, R.D. Field, M. Fisher, I.K. Furic, J. Hugon, J. Konigsberg, A. Korytov, T. Kypreos, J.F. Low, K. Matchev, P. Milenovic ${ }^{49}$, G. Mitselmakher, L. Muniz, A. Rinkevicius, L. Shchutska, M. Snowball, D. Sperka, J. Yelton, M. Zakaria

Florida International University, Miami, U.S.A.

S. Hewamanage, S. Linn, P. Markowitz, G. Martinez, J.L. Rodriguez

Florida State University, Tallahassee, U.S.A.

T. Adams, A. Askew, J. Bochenek, B. Diamond, J. Haas, S. Hagopian, V. Hagopian, K.F. Johnson, H. Prosper, V. Veeraraghavan, M. Weinberg

Florida Institute of Technology, Melbourne, U.S.A.

M.M. Baarmand, M. Hohlmann, H. Kalakhety, F. Yumiceva 
University of Illinois at Chicago (UIC), Chicago, U.S.A.

M.R. Adams, L. Apanasevich, V.E. Bazterra, D. Berry, R.R. Betts, I. Bucinskaite, R. Cavanaugh, O. Evdokimov, L. Gauthier, C.E. Gerber, D.J. Hofman, S. Khalatyan, P. Kurt, D.H. Moon, C. O'Brien, C. Silkworth, P. Turner, N. Varelas

The University of Iowa, Iowa City, U.S.A.

E.A. Albayrak ${ }^{50}$, B. Bilki ${ }^{51}$, W. Clarida, K. Dilsiz, F. Duru, M. Haytmyradov, J.-P. Merlo, H. Mermerkaya ${ }^{52}$, A. Mestvirishvili, A. Moeller, J. Nachtman, H. Ogul, Y. Onel, F. Ozok ${ }^{50}$, A. Penzo, R. Rahmat, S. Sen, P. Tan, E. Tiras, J. Wetzel, T. Yetkin ${ }^{53}$, K. Yi

Johns Hopkins University, Baltimore, U.S.A.

B.A. Barnett, B. Blumenfeld, S. Bolognesi, D. Fehling, A.V. Gritsan, P. Maksimovic, C. Martin, M. Osherson, M. Swartz, Y. Xin

The University of Kansas, Lawrence, U.S.A.

P. Baringer, A. Bean, G. Benelli, C. Bruner, R.P. Kenny III, M. Malek, M. Murray, D. Noonan, S. Sanders, J. Sekaric, R. Stringer, Q. Wang, J.S. Wood

Kansas State University, Manhattan, U.S.A.

A.F. Barfuss, I. Chakaberia, A. Ivanov, S. Khalil, M. Makouski, Y. Maravin, L.K. Saini, S. Shrestha, N. Skhirtladze, I. Svintradze

Lawrence Livermore National Laboratory, Livermore, U.S.A.

J. Gronberg, D. Lange, F. Rebassoo, D. Wright

University of Maryland, College Park, U.S.A.

A. Baden, A. Belloni, B. Calvert, S.C. Eno, J.A. Gomez, N.J. Hadley, R.G. Kellogg,

T. Kolberg, Y. Lu, M. Marionneau, A.C. Mignerey, K. Pedro, A. Skuja, M.B. Tonjes, S.C. Tonwar

Massachusetts Institute of Technology, Cambridge, U.S.A.

A. Apyan, R. Barbieri, G. Bauer, W. Busza, I.A. Cali, M. Chan, L. Di Matteo, V. Dutta, G. Gomez Ceballos, M. Goncharov, D. Gulhan, M. Klute, Y.S. Lai, Y.-J. Lee, A. Levin, P.D. Luckey, T. Ma, C. Paus, D. Ralph, C. Roland, G. Roland, G.S.F. Stephans, F. Stöckli, K. Sumorok, D. Velicanu, J. Veverka, B. Wyslouch, M. Yang, M. Zanetti, V. Zhukova

University of Minnesota, Minneapolis, U.S.A.

B. Dahmes, A. Gude, S.C. Kao, K. Klapoetke, Y. Kubota, J. Mans, N. Pastika, R. Rusack, A. Singovsky, N. Tambe, J. Turkewitz

University of Mississippi, Oxford, U.S.A.

J.G. Acosta, S. Oliveros

University of Nebraska-Lincoln, Lincoln, U.S.A.

E. Avdeeva, K. Bloom, S. Bose, D.R. Claes, A. Dominguez, R. Gonzalez Suarez, J. Keller,

D. Knowlton, I. Kravchenko, J. Lazo-Flores, S. Malik, F. Meier, G.R. Snow

State University of New York at Buffalo, Buffalo, U.S.A.

J. Dolen, A. Godshalk, I. Iashvili, A. Kharchilava, A. Kumar, S. Rappoccio 
Northeastern University, Boston, U.S.A.

G. Alverson, E. Barberis, D. Baumgartel, M. Chasco, J. Haley, A. Massironi, D.M. Morse,

D. Nash, T. Orimoto, D. Trocino, R.-J. Wang, D. Wood, J. Zhang

Northwestern University, Evanston, U.S.A.

K.A. Hahn, A. Kubik, N. Mucia, N. Odell, B. Pollack, A. Pozdnyakov, M. Schmitt, S. Stoynev, K. Sung, M. Velasco, S. Won

University of Notre Dame, Notre Dame, U.S.A.

A. Brinkerhoff, K.M. Chan, A. Drozdetskiy, M. Hildreth, C. Jessop, D.J. Karmgard, N. Kellams, K. Lannon, W. Luo, S. Lynch, N. Marinelli, T. Pearson, M. Planer, R. Ruchti, N. Valls, M. Wayne, M. Wolf, A. Woodard

The Ohio State University, Columbus, U.S.A.

L. Antonelli, J. Brinson, B. Bylsma, L.S. Durkin, S. Flowers, C. Hill, R. Hughes, K. Kotov, T.Y. Ling, D. Puigh, M. Rodenburg, G. Smith, B.L. Winer, H. Wolfe, H.W. Wulsin

Princeton University, Princeton, U.S.A.

O. Driga, P. Elmer, P. Hebda, A. Hunt, S.A. Koay, P. Lujan, D. Marlow, T. Medvedeva, M. Mooney, J. Olsen, P. Piroué, X. Quan, H. Saka, D. Stickland ${ }^{2}$, C. Tully, J.S. Werner, A. Zuranski

University of Puerto Rico, Mayaguez, U.S.A.

E. Brownson, H. Mendez, J.E. Ramirez Vargas

Purdue University, West Lafayette, U.S.A.

V.E. Barnes, D. Benedetti, G. Bolla, D. Bortoletto, M. De Mattia, Z. Hu, M.K. Jha, M. Jones, K. Jung, M. Kress, N. Leonardo, D. Lopes Pegna, V. Maroussov, P. Merkel, D.H. Miller, N. Neumeister, B.C. Radburn-Smith, X. Shi, I. Shipsey, D. Silvers, A. Svyatkovskiy, F. Wang, W. Xie, L. Xu, H.D. Yoo, J. Zablocki, Y. Zheng

Purdue University Calumet, Hammond, U.S.A.

N. Parashar, J. Stupak

Rice University, Houston, U.S.A.

A. Adair, B. Akgun, K.M. Ecklund, F.J.M. Geurts, W. Li, B. Michlin, B.P. Padley, R. Redjimi, J. Roberts, J. Zabel

University of Rochester, Rochester, U.S.A.

B. Betchart, A. Bodek, R. Covarelli, P. de Barbaro, R. Demina, Y. Eshaq, T. Ferbel, A. Garcia-Bellido, P. Goldenzweig, J. Han, A. Harel, A. Khukhunaishvili, G. Petrillo, D. Vishnevskiy

The Rockefeller University, New York, U.S.A.

R. Ciesielski, L. Demortier, K. Goulianos, G. Lungu, C. Mesropian

Rutgers, The State University of New Jersey, Piscataway, U.S.A.

S. Arora, A. Barker, J.P. Chou, C. Contreras-Campana, E. Contreras-Campana, D. Duggan, D. Ferencek, Y. Gershtein, R. Gray, E. Halkiadakis, D. Hidas, S. Kaplan, A. Lath, 
S. Panwalkar, M. Park, R. Patel, S. Salur, S. Schnetzer, S. Somalwar, R. Stone, S. Thomas, P. Thomassen, M. Walker

University of Tennessee, Knoxville, U.S.A.

K. Rose, S. Spanier, A. York

\section{Texas A\&M University, College Station, U.S.A.}

O. Bouhali ${ }^{54}$, A. Castaneda Hernandez, R. Eusebi, W. Flanagan, J. Gilmore, T. Kamon ${ }^{55}$, V. Khotilovich, V. Krutelyov, R. Montalvo, I. Osipenkov, Y. Pakhotin, A. Perloff, J. Roe, A. Rose, A. Safonov, T. Sakuma, I. Suarez, A. Tatarinov

\section{Texas Tech University, Lubbock, U.S.A.}

N. Akchurin, C. Cowden, J. Damgov, C. Dragoiu, P.R. Dudero, J. Faulkner, K. Kovitanggoon, S. Kunori, S.W. Lee, T. Libeiro, I. Volobouev

Vanderbilt University, Nashville, U.S.A.

E. Appelt, A.G. Delannoy, S. Greene, A. Gurrola, W. Johns, C. Maguire, Y. Mao, A. Melo, M. Sharma, P. Sheldon, B. Snook, S. Tuo, J. Velkovska

University of Virginia, Charlottesville, U.S.A.

M.W. Arenton, S. Boutle, B. Cox, B. Francis, J. Goodell, R. Hirosky, A. Ledovskoy, H. Li, C. Lin, C. Neu, J. Wood

Wayne State University, Detroit, U.S.A.

C. Clarke, R. Harr, P.E. Karchin, C. Kottachchi Kankanamge Don, P. Lamichhane, J. Sturdy

\section{University of Wisconsin, Madison, U.S.A.}

D.A. Belknap, D. Carlsmith, M. Cepeda, S. Dasu, L. Dodd, S. Duric, E. Friis, R. HallWilton, M. Herndon, A. Hervé, P. Klabbers, A. Lanaro, C. Lazaridis, A. Levine, R. Loveless, A. Mohapatra, I. Ojalvo, T. Perry, G.A. Pierro, G. Polese, I. Ross, T. Sarangi, A. Savin, W.H. Smith, D. Taylor, C. Vuosalo, N. Woods

$\dagger$ : Deceased

1: Also at Vienna University of Technology, Vienna, Austria

2: Also at CERN, European Organization for Nuclear Research, Geneva, Switzerland

3: Also at Institut Pluridisciplinaire Hubert Curien, Université de Strasbourg, Université de Haute Alsace Mulhouse, CNRS/IN2P3, Strasbourg, France

4: Also at National Institute of Chemical Physics and Biophysics, Tallinn, Estonia

5: Also at Skobeltsyn Institute of Nuclear Physics, Lomonosov Moscow State University, Moscow, Russia

6: Also at Universidade Estadual de Campinas, Campinas, Brazil

7: Also at Laboratoire Leprince-Ringuet, Ecole Polytechnique, IN2P3-CNRS, Palaiseau, France

8: Also at Joint Institute for Nuclear Research, Dubna, Russia

9: Also at Suez University, Suez, Egypt

10: Also at Cairo University, Cairo, Egypt

11: Also at Fayoum University, El-Fayoum, Egypt 
12: Also at British University in Egypt, Cairo, Egypt

13: Now at Sultan Qaboos University, Muscat, Oman

14: Also at Université de Haute Alsace, Mulhouse, France

15: Also at Brandenburg University of Technology, Cottbus, Germany

16: Also at Institute of Nuclear Research ATOMKI, Debrecen, Hungary

17: Also at Eötvös Loránd University, Budapest, Hungary

18: Also at University of Debrecen, Debrecen, Hungary

19: Also at University of Visva-Bharati, Santiniketan, India

20: Now at King Abdulaziz University, Jeddah, Saudi Arabia

21: Also at University of Ruhuna, Matara, Sri Lanka

22: Also at Isfahan University of Technology, Isfahan, Iran

23: Also at Sharif University of Technology, Tehran, Iran

24: Also at Plasma Physics Research Center, Science and Research Branch, Islamic Azad University, Tehran, Iran

25: Also at Università degli Studi di Siena, Siena, Italy

26: Also at Centre National de la Recherche Scientifique (CNRS) - IN2P3, Paris, France

27: Also at Purdue University, West Lafayette, U.S.A.

28: Also at Universidad Michoacana de San Nicolas de Hidalgo, Morelia, Mexico

29: Also at Institute for Nuclear Research, Moscow, Russia

30: Also at St. Petersburg State Polytechnical University, St. Petersburg, Russia

31: Also at California Institute of Technology, Pasadena, U.S.A.

32: Also at INFN Sezione di Padova; Università di Padova; Università di Trento (Trento), Padova, Italy

33: Also at Faculty of Physics, University of Belgrade, Belgrade, Serbia

34: Also at Facoltà Ingegneria, Università di Roma, Roma, Italy

35: Also at Scuola Normale e Sezione dell'INFN, Pisa, Italy

36: Also at University of Athens, Athens, Greece

37: Also at Paul Scherrer Institut, Villigen, Switzerland

38: Also at Institute for Theoretical and Experimental Physics, Moscow, Russia

39: Also at Albert Einstein Center for Fundamental Physics, Bern, Switzerland

40: Also at Gaziosmanpasa University, Tokat, Turkey

41: Also at Adiyaman University, Adiyaman, Turkey

42: Also at Cag University, Mersin, Turkey

43: Also at Izmir Institute of Technology, Izmir, Turkey

44: Also at Ozyegin University, Istanbul, Turkey

45: Also at Marmara University, Istanbul, Turkey

46: Also at Kafkas University, Kars, Turkey

47: Also at Rutherford Appleton Laboratory, Didcot, United Kingdom

48: Also at School of Physics and Astronomy, University of Southampton, Southampton, United Kingdom

49: Also at University of Belgrade, Faculty of Physics and Vinca Institute of Nuclear Sciences, Belgrade, Serbia

50: Also at Mimar Sinan University, Istanbul, Istanbul, Turkey

51: Also at Argonne National Laboratory, Argonne, U.S.A.

52: Also at Erzincan University, Erzincan, Turkey

53: Also at Yildiz Technical University, Istanbul, Turkey

54: Also at Texas A\&M University at Qatar, Doha, Qatar

55: Also at Kyungpook National University, Daegu, Korea 